\title{
Chromatic Polynomials for Lattice Strips with Cyclic Boundary Conditions
}

\author{
Shu-Chiuan Chang* \\ C. N. Yang Institute for Theoretical Physics \\ State University of New York \\ Stony Brook, N. Y. 11794-3840 \\ $U S A$
}

\begin{abstract}
The zero-temperature $q$-state Potts model partition function for a lattice strip of fixed width $L_{y}$ and arbitrary length $L_{x}$ has the form $P(G, q)=$ $\sum_{j=1}^{N_{G, \lambda}} c_{G, j}\left(\lambda_{G, j}\right)^{L_{x}}$, and is equivalent to the chromatic polynomial for this graph. We present exact zero-temperature partition functions for strips of several lattices with $\left(F B C_{y}, P B C_{x}\right)$, i.e., cyclic, boundary conditions. In particular, the chromatic polynomial of a family of generalized dodecahedra graphs is calculated. The coefficient $c_{G, j}$ of degree $d$ in $q$ is $c^{(d)}=U_{2 d}\left(\frac{\sqrt{q}}{2}\right)$, where $U_{n}(x)$ is the Chebyshev polynomial of the second kind. We also present the chromatic polynomial for the strip of the square lattice with $\left(P B C_{y}, P B C_{x}\right)$, i.e., toroidal, boundary conditions and width $L_{y}=4$ with the property that each set of four vertical vertices forms a tetrahedron. A number of interesting and novel features of the continuous accumulation set of the chromatic zeros, $\mathcal{B}$ are found.
\end{abstract}

*email: shu-chiuan.chang@sunysb.edu 


\section{INTRODUCTION}

The $q$-state Potts antiferromagnet (AF) [1.2] exhibits nonzero ground state entropy, $S_{0}>$ 0 (without frustration) for sufficiently large $q$ on a given lattice $\Lambda$ or, more generally, on a graph $G$. This is equivalent to a ground state degeneracy per site $W>1$, since $S_{0}=k_{B} \ln W$. There is a close connection with graph theory here, since the zero-temperature partition function of the above-mentioned $q$-state Potts antiferromagnet on a graph $G$ satisfies

$$
Z(G, q, T=0)_{P A F}=P(G, q),
$$

where $P(G, q)$ is the chromatic polynomial expressing the number of ways of coloring the vertices of the graph $G$ with $q$ colors such that no two adjacent vertices have the same color (for reviews, see [3]- [5]). The minimum number of colors necessary for such a coloring of $G$ is called the chromatic number, $\chi(G)$. Thus

$$
W(\{G\}, q)=\lim _{n \rightarrow \infty} P(G, q)^{1 / n},
$$

where $n$ is the number of vertices of $G$ and $\{G\}=\lim _{n \rightarrow \infty} G$. Where no confusion will result, we shall sometimes write $G$ rather than $\{G\}$ for the infinite-length limit of a given type of strip graph. At certain special points $q_{s}$ (typically $q_{s}=0,1, . ., \chi(G)$ ), one has the noncommutativity of limits

$$
\lim _{q \rightarrow q_{s}} \lim _{n \rightarrow \infty} P(G, q)^{1 / n} \neq \lim _{n \rightarrow \infty} \lim _{q \rightarrow q_{s}} P(G, q)^{1 / n},
$$

and hence it is necessary to specify the order of the limits in the definition of $W\left(\{G\}, q_{s}\right)$ [6]. Denoting $W_{q n}$ and $W_{n q}$ as the functions defined by the different order of limits on the left and right-hand sides of (1.3), we take $W \equiv W_{q n}$ here; this has the advantage of removing certain isolated discontinuities that are present in $W_{n q}$.

Using the expression for $P(G, q)$, one can generalize $q$ from $\mathbb{Z}_{+}$to $\mathbb{C}$. The zeros of $P(G, q)$ in the complex $q$ plane are called chromatic zeros; a subset of these may form an accumulation set in the $n \rightarrow \infty$ limit, denoted $\mathcal{B}$, which is the continuous locus of points where $W(\{G\}, q)$ is nonanalytic. The maximal region in the complex $q$ plane to which one can analytically continue the function $W(\{G\}, q)$ from physical values where there is nonzero ground state entropy is denoted $R_{1}$. The maximal value of $q$ where $\mathcal{B}$ intersects the (positive) real axis is labeled $q_{c}(\{G\})$. This point is important since $W(\{G\}, q)$ is a real analytic function from large values of $q$ down to $q_{c}(\{G\})$.

\footnotetext{
${ }^{1}$ For some families of graphs $\mathcal{B}$ may be null, and $W$ may also be nonanalytic at certain discrete points.
} 


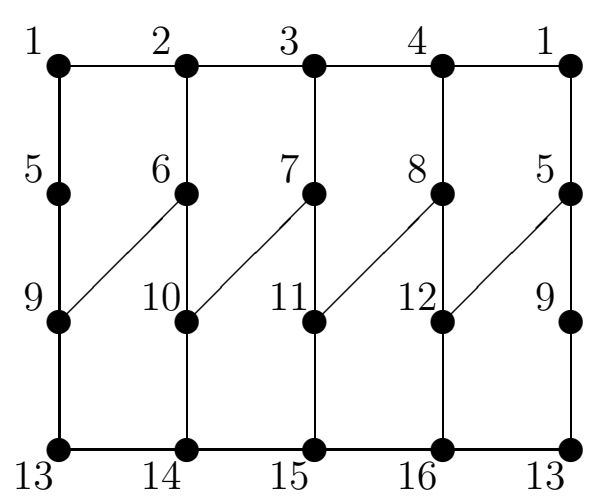

(a)

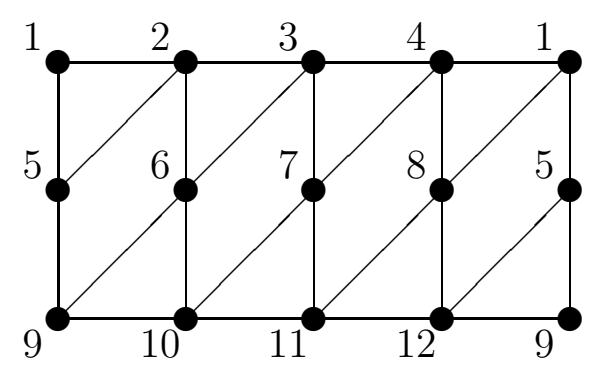

(c)

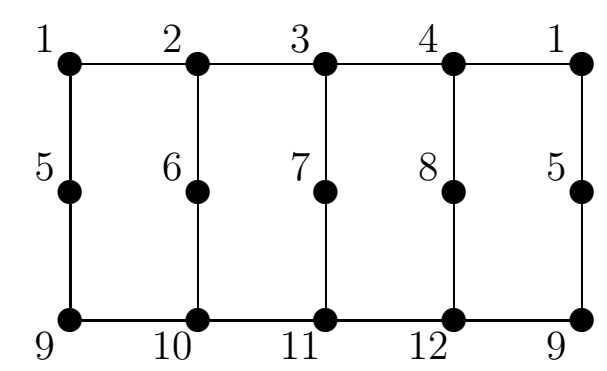

(e)

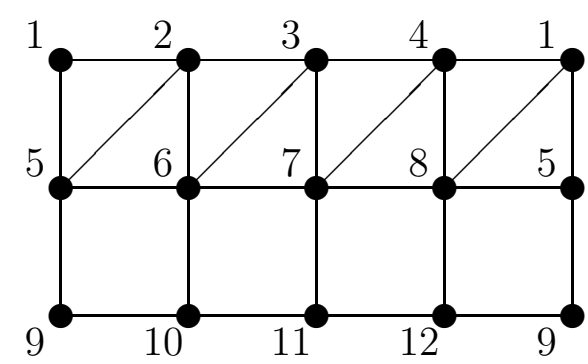

(b)

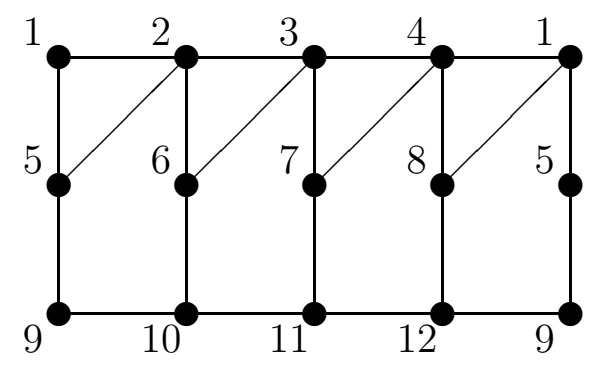

(d)

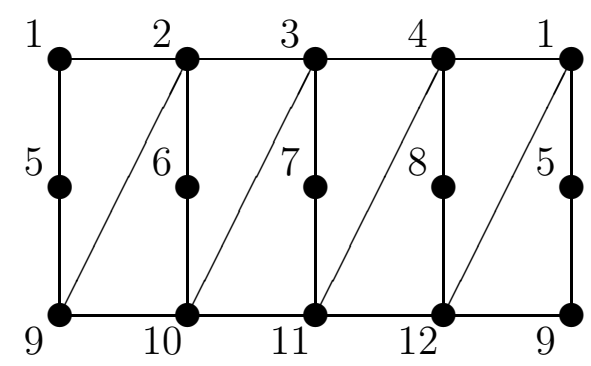

(f)

FIG. 1. Illustrative strip graphs of the following lattices with length $L_{x}=m=4$ and $\left(F B C_{y}, P B C_{x}\right)$ boundary condition (cyclic): (a) $O_{m}$ lattice with $L_{y}=4$ (b) $Q_{m}$ lattice with $L_{y}=3$ (c) $S_{m}$ lattice with $L_{y}=3$ (d) $V_{m}$ lattice with $L_{y}=3$ (e) $X_{k, m}$ lattice with $k=3$ (f) $Y_{k, m}$ lattice with $k=3$.

In the present work we report exact solutions for chromatic polynomials $P(G, q)$ for strips of lattices with arbitrarily great length $L_{x}$ vertices and finite width $L_{y}$. Consider $L_{x}$ sets of disjoint tree graphs, $T_{L_{y}}$ with vertex set $v\left(T_{L_{y}}\right)=\left\{1,2, \ldots, L_{y}\right\}$. Denote the edges joining each adjacent pair of $T_{L_{y}}$ and $T_{L_{y}}^{\prime}$ graphs as $e=\left\{v_{i} v_{j}^{\prime} \mid i, j=1, \ldots, L_{y}\right\}$, where $v_{i}, v_{j}^{\prime}$ are vertices of $T_{L_{y}}$ and $T_{L_{y}}^{\prime}$. Impose periodic boundary conditions in the longitudinal direction, 
denoted $x$. The lattices in which we are interested here are (a) $L_{y}=4$ and $e=\{11,32,44\}$; (b) $L_{y}=3$ and $e=\{11,21,22,33\}$; (c) $L_{y}=3$ and $e=\{11,21,32,33\}$; (d) $L_{y}=3$ and $e=\{11,21,33\}$; (e) $L_{y}=k$ and $e=\{11, k k\}$; (f) $L_{y}=k$ and $e=\{11, k 1, k k\}$. These families of graphs will be denoted as $O_{m}, Q_{m}, S_{m}, V_{m}, X_{k, m}, Y_{k, m}$ in the present work, where $m=L_{x}$. Illustrative examples for these lattices with $m=4$ are displayed in Fig. 1. The total number of vertices is $L_{x} L_{y}$ for all these strips. We have given the chromatic polynomial for the $L_{y}=3$ triangular lattice with $e=\{11,21,22,32,33\}$ and cyclic boundary condition before [7], and $Q_{m}, S_{m}$, and $V_{m}$ here are the corresponding lattice strips without one of the diagonal edges, without the middle horizontal edge, and without both of these edges in each subgraph, respectively. $X_{k, m}$ and $Y_{k, m}$ come from the homeomorphic expansions on the vertical edges of the cyclic $L_{y}=2$ strips of the square and triangular lattices.

A generic form for chromatic polynomials for recursively defined families of graphs, of which strip graphs $G_{s}$ are special cases, is [B]

$$
P\left(\left(G_{s}\right)_{m}, q\right)=\sum_{j=1}^{N_{G_{s}, \lambda}} c_{G_{s}, j}(q)\left(\lambda_{G_{s}, j}(q)\right)^{m}
$$

where $c_{G_{s}, j}(q)$ and the $N_{G_{s}, \lambda}$ terms $\lambda_{G_{s}, j}(q)$ depend on the type of strip graph $G_{s}$ but are independent of $m$.

The coefficients $c_{G_{s}, j}(q)$ that enter into the expressions for the chromatic polynomial (1.4) for the cyclic strip of the families of lattice considered here are

$$
c^{(d)}=U_{2 d}\left(\frac{\sqrt{q}}{2}\right)
$$

where $U_{n}(x)$ is the Chebyshev polynomial of the second kind, defined by

$$
U_{n}(x)=\sum_{j=0}^{\left[\frac{n}{2}\right]}(-1)^{j}\left(\begin{array}{c}
n-j \\
j
\end{array}\right)(2 x)^{n-2 j}
$$

as we found for the cyclic strips of square and triangular lattices [9]. Here in eq. (1.6) the notation $\left[\frac{n}{2}\right]$ in the upper limit on the summand means the integral part of $\frac{n}{2}$. The first few of these coefficients are

$$
\begin{gathered}
c^{(0)}=1, \quad c^{(1)}=q-1, \\
c^{(2)}=q^{2}-3 q+1,
\end{gathered}
$$

and 


$$
c^{(3)}=q^{3}-5 q^{2}+6 q-1 .
$$

When the coefficient $c^{(0)}$ appears in chromatic polynomials, we shall simply write it as unity.

For a given type of strip graph $G_{s}$, we denote the sum of the coefficients $c_{G_{s}, j}$ as

$$
C\left(G_{s}\right)=\sum_{j=1}^{N_{G_{s}, \lambda}} c_{G_{s}, j} .
$$

It will also be convenient to define the following polynomial:

$$
D_{k}(q)=\frac{P\left(C_{k}, q\right)}{q(q-1)}=\sum_{s=0}^{k-2}(-1)^{s}\left(\begin{array}{c}
k-1 \\
s
\end{array}\right) q^{k-2-s}
$$

where $P\left(C_{n}, q\right)=(q-1)^{n}+(q-1)(-1)^{n}$ is the chromatic polynomial for the circuit (cyclic) graph $C_{n}$ with $n$ vertices. Some works on calculations of chromatic polynomials for recursive families of graphs include [1]- 45].

\section{FAMILY OF $O_{M}$ STRIPS WITH $\left(F B C_{Y}, P B C_{X}\right)$}

In this section we give our solutions for the chromatic polynomials of the $4 \times m$ strips of the $O_{m}$ lattice strip with cyclic boundary conditions. The chromatic number for this family is $\chi(O)=3$. We calculate the chromatic polynomials by iterated use of the deletion-contraction theorem, via a generating function approach [22,24], and a coloring matrix method [13]. We find $N_{O, \lambda}=14$ and

$$
P\left(O_{m}, F B C_{y}, P B C_{x}, q\right)=\sum_{j=1}^{14} c_{O, j}\left(\lambda_{O, j}\right)^{m}
$$

where $\lambda_{O, j}$ 's for $1 \leq j \leq 13$ are roots of a cubic equation, a sixth-degree equation, and a quartic equation. Specifically, the $\lambda_{O, j}$ 's for $1 \leq j \leq 3$ are roots of the equation

$$
\begin{aligned}
& \xi^{3}-\left(q^{4}-6 q^{3}+16 q^{2}-24 q+17\right) \xi^{2} \\
& +(q-1)(q-2)\left(q^{4}-7 q^{3}+20 q^{2}-28 q+18\right) \xi-(q-1)^{2}(q-2)^{2}=0
\end{aligned}
$$

while the $\lambda_{O, j}$ 's for $4 \leq j \leq 9$ are roots of the equation

$\xi^{6}+2\left(q^{3}-6 q^{2}+14 q-13\right) \xi^{5}+\left(q^{2}-5 q+7\right)\left(q^{4}-9 q^{3}+30 q^{2}-47 q+29\right) \xi^{4}$ 


$$
\begin{aligned}
& -\left(2 q^{8}-30 q^{7}+202 q^{6}-796 q^{5}+2001 q^{4}-3278 q^{3}+3420 q^{2}-2084 q+567\right) \xi^{3} \\
& +(q-1)\left(q^{9}-17 q^{8}+130 q^{7}-586 q^{6}+1717 q^{5}-3401 q^{4}+4578 q^{3}-4072 q^{2}+2199 q-557\right) \xi^{2} \\
& -2(q-1)^{2}(q-2)\left(q^{5}-9 q^{4}+33 q^{3}-61 q^{2}+57 q-23\right) \xi \\
& +(q-1)^{4}(q-2)^{2}=0
\end{aligned}
$$

the $\lambda_{O, j}$ 's for $10 \leq j \leq 13$ are roots of the equation

$$
\begin{aligned}
& \xi^{4}-\left(q^{2}-6 q+10\right) \xi^{3}-\left(2 q^{3}-13 q^{2}+30 q-23\right) \xi^{2} \\
& -(q-1)(q-3)\left(q^{2}-4 q+5\right) \xi+(q-1)^{2}=0
\end{aligned}
$$

and finally, $\lambda_{O, 14}=1$.

The corresponding coefficients are

$$
\begin{gathered}
c_{O, j}=1 \quad \text { for } \quad 1 \leq j \leq 3 \\
c_{O, j}=c^{(1)} \quad \text { for } \quad 4 \leq j \leq 9 \\
c_{O, j}=c^{(2)} \text { for } 10 \leq j \leq 13
\end{gathered}
$$

and

$$
c_{O, 14}=c^{(3)}
$$

Here the coefficients have the form of the Chebyshev polynomial of the second kind given in (1.6)-(1.9) . There is also a zero eigenvalue $\lambda_{O, 15}=0$ with coefficient $c_{O, 15}=q(q-1)\left(q^{2}-\right.$ $3 q+1$ ), which does not contribute to the chromatic polynomial (2.1). The sum of all of the coefficients is equal to $P\left(T_{4}, q\right)=q(q-1)^{3}$ if we include $c_{O, 15}$, and is equal to $q^{2}(q-1)$ if $c_{O, 15}$ is not included.

The locus $\mathcal{B}$ separates the $q$ plane into three main regions. The outermost one, region $R_{1}$, extends to infinite $|q|$ and includes the intervals $q \geq q_{c}$ and $q \leq 0$ on the real $q$ axis. Here,

$$
q_{c}\left(O, F B C_{y}, P B C_{x}\right) \simeq 2.638342
$$


Region $R_{2}$ includes the real interval $2 \leq q \leq q_{c}$, while region $R_{3}$ includes the real interval $0 \leq q \leq 2$. In regions $R_{i}, 1 \leq i \leq 3$, the dominant terms are, respectively, the root with maximal magnitude of (1) the cubic equation (2.2), (2) the quartic equation (2.4), (3) the sixth-degree equation (2.3). Our previous calculations for various families of graphs [30,7] have shown that $\mathcal{B}$ can include pairs of extremely small complex-conjugate regions. We have not made an exhaustive search for these in the present case. The locus $\mathcal{B}$ for this family (defined in the limit $n \rightarrow \infty$ ) and, for comparison, chromatic zeros for a typical long finite $O_{m}$ strip, with $L_{x}=m=20$, are shown in Fig. 2. With this value of $m$, the chromatic zeros lie close to the boundary $\mathcal{B}$. The locus $\mathcal{B}$ crosses the real $q$-axis at $q=0,2$ and $q_{c}$.

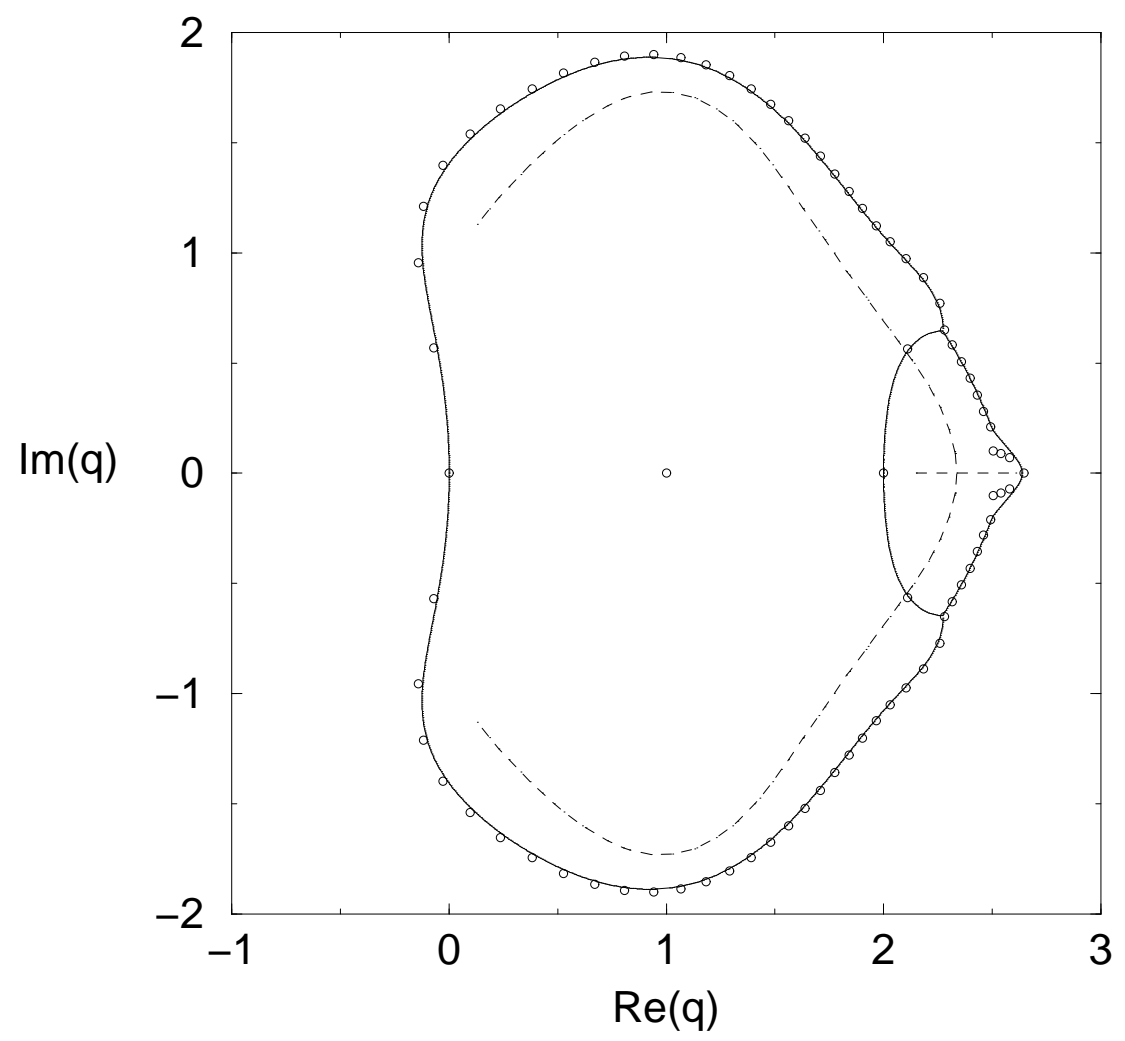


FIG. 2. The locus $\mathcal{B}$ for the $m \rightarrow \infty$ limit of the $O_{m}$ family of cyclic strip graphs (solid curve). For comparison, we show chromatic zeros for the cyclic $O_{m}$ strip graph with $m=20$, i.e., $n=80$ vertices. The dashed line is the locus of solutions of the degeneracy equation involving only the roots of the cubic eq. (2.2).

Earlier, it was shown that the locus $\mathcal{B}$ can have support for $\operatorname{Re}(q) \leq 0$ in 30 for square lattice strips and in [0] for triangular lattice with cyclic or Möbius boundary conditions. These families have respective girths equal to 4 and 3, respectively, for $L_{x}$ large enough to avoid degenerate cases. Chromatic zeros with negative real parts were also found for several other families of graphs [17,24], including those that yielded chromatic zeros with arbitrarily large magnitude $|q|[26,33]$. Here we find that $\mathcal{B}(O)$ also has support for $\operatorname{Re}(q) \leq 0$ for the present $m \rightarrow \infty$ limit of) the $O_{m}$ family, which has girth 5 . Although the chromatic roots of regular dodecahedron, denoted as $\mathrm{O}_{5}$ here, does not have any negative real part, where the smallest one is about 0.0082126 , except for the trivial root $q=0$, the chromatic roots of $O_{m}$ for $m \geq 6$ do include some with negative real parts. The largest magnitude of the negative real part increases as $m$ increases, and approaches the limit of $\mathcal{B}$ up to $q \simeq-0.125$. The density of zeros is observed to be relatively small on the parts of $\mathcal{B}$ extending through $q=0$ and 2 , separating $R_{3}$ from $R_{1}$ and $R_{2}$ near the real $q$-axis.

Partial results for the chromatic polynomial of the $O_{m}$ family were reported in [44], where this family was denoted as $D_{n}$. Where they overlap, some of the results in our complete calculation differ with those in [44] 46].

\section{FAMILY OF $Q_{M}$ STRIPS WITH $\left(F B C_{Y}, P B C_{X}\right)$}

In this section we consider the chromatic polynomials of the $3 \times m$ strips of the $Q_{m}$ lattice with $\left(F B C_{y}, P B C_{x}\right)$, i.e. cyclic, boundary conditions. This family of graphs has one more diagonal edge in each subgraph than the $L_{y}=3$ square lattice with cyclic boundary condition [29], and one less diagonal edge than the $L_{y}=3$ triangular lattice with the same boundary condition [7]. We have $N_{Q, \lambda}=10$, and

$$
P\left(Q_{m}, F B C_{y}, P B C_{x}, q\right)=\sum_{j=1}^{10} c_{Q, j}\left(\lambda_{Q, j}\right)^{m}
$$

where

$$
\lambda_{Q,(1,2)}=\frac{1}{2}\left(q^{3}-6 q^{2}+14 q-13 \pm \sqrt{(q-3)\left(q^{2}-5 q+7\right)\left(q^{3}-4 q^{2}+6 q-5\right)}\right) .
$$

The $\lambda_{Q, j}$ for $3 \leq j \leq 6$ are the roots of the quartic equation 


$$
\begin{gathered}
\xi^{4}+\left(3 q^{2}-15 q+20\right) \xi^{3}+\left(3 q^{4}-30 q^{3}+114 q^{2}-191 q+117\right) \xi^{2} \\
+(q-2)\left(q^{5}-14 q^{4}+76 q^{3}-199 q^{2}+251 q-121\right) \xi-(q-2)^{5}\left(q^{2}-5 q+5\right)=0 \\
\lambda_{Q,(7,8)}=q-3 \pm \sqrt{4-q}
\end{gathered}
$$

and $\lambda_{Q, 9}=q-2, \lambda_{Q, 10}=-1$. The coefficients are

$$
\begin{gathered}
c_{Q, j}=1 \quad \text { for } \quad 1 \leq j \leq 2 \\
c_{Q, j}=c^{(1)} \text { for } 3 \leq j \leq 6 \\
c_{Q, j}=c^{(2)} \text { for } 7 \leq j \leq 9
\end{gathered}
$$

and

$$
c_{Q, 10}=c^{(3)} .
$$

Again only one type of polynomial of each degree in $q$ occurs in the coefficients, and the number of $\lambda$ 's with coefficients $c^{(d)}$, which is denoted as $n_{P}\left(L_{y}, d\right)$ in [9], are exactly the same as those for the square and triangular lattices with $L_{y}=3: n_{P}(3,0)=2, n_{P}(3,1)=$ $4, n_{P}(3,2)=3, n_{P}(3,3)=1$. Therefore, the sum of the coefficients is

$$
C\left(Q, F B C_{y}, P B C_{x}\right)=\sum_{j=1}^{10} c_{Q, j}=q(q-1)^{2} .
$$

For the family $Q_{m}$ of lattice strips, we find that $q_{c}=3$; at this point all the $\lambda_{Q}$ 's have the same magnitude 1 . The locus $\mathcal{B}$ crosses the real $q$-axis at $q=0,2$ and $q_{c}=3$, and separates the $q$ plane into five main regions. Region $R_{1}$ extends to infinite $|q|$ and includes the intervals $q \geq 3$ and $q \leq 0$ on the real $q$ axis. Region $R_{2}$ includes the real interval $2 \leq q \leq 3$, and region $R_{3}$ includes the real interval $0 \leq q \leq 2$. There is also a pair of complex-conjugate regions $R_{4}, R_{4}^{*}$ centered at approximately $q \simeq 2.55 \pm 1.30 i$. We have not made an exhaustive search for the tiny regions in this case. The dominant terms in regions $R_{1}$ and $R_{2}$ are $\lambda_{Q, 1}$ in (3.2) and $\lambda_{Q, 7}$ in (3.4), while regions $R_{3}$ and $R_{4}, R_{4}^{*}$ are both dominated by the roots of the quartic equation (3.3) with maximal magnitude. The locus $\mathcal{B}$ has support for $\operatorname{Re}(q) \geq 0$, and the density of zeros is smaller on the parts of $\mathcal{B}$ extending through $q=0$ and 2 , separating $R_{3}$ from $R_{1}$ and $R_{2}$.

The locus $\mathcal{B}$ and chromatic zeros for the $L_{x}=m=20$ cyclic graph of the $Q_{m}$ lattice are shown in Fig. 3. The chromatic zeros lie close to the boundary $\mathcal{B}$ and indicate its position. 


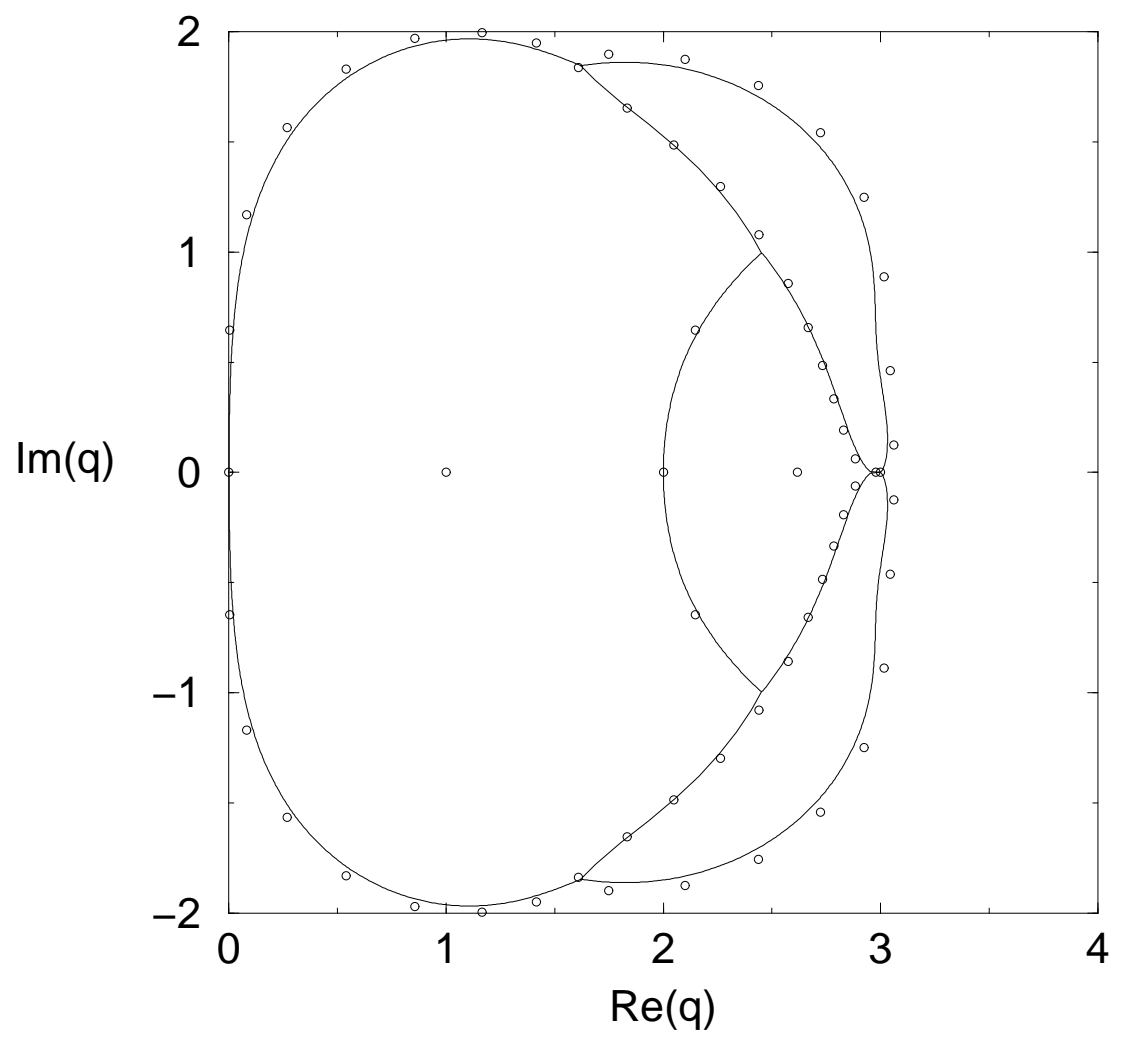

FIG. 3. Locus $\mathcal{B}$ for the $m \rightarrow \infty$ limit of the family of cyclic graphs $Q_{m}$. For comparison, chromatic zeros for $Q_{m}$ with $m=20$ (i.e., $n=60$ ) are shown.

\section{FAMILY OF $S_{M}$ STRIPS WITH $\left(F B C_{Y}, P B C_{X}\right)$}

The $3 \times m$ strips of the $S_{m}$ lattice with $\left(F B C_{y}, P B C_{x}\right)=$ cyclic boundary conditions have one less middle horizontal edge in each subgraph than the $L_{y}=3$ triangular lattice with the same boundary conditions [0]. For the solutions of the chromatic polynomial, we find $N_{S, \lambda}=6$ and

$$
P\left(S_{m}, F B C_{y}, P B C_{x}, q\right)=\sum_{j=1}^{6} c_{S, j}\left(\lambda_{S, j}\right)^{m},
$$


where

$$
\begin{gathered}
\lambda_{S,(1,2)}=\frac{q-2}{2}\left(q^{2}-4 q+6 \pm \sqrt{q^{4}-8 q^{3}+24 q^{2}-36 q+28}\right) \\
\lambda_{S,(3,4)}=\frac{-1}{2}\left((q-3)(q-4) \pm \sqrt{q^{4}-10 q^{3}+49 q^{2}-120 q+112}\right),
\end{gathered}
$$

and $\lambda_{S, 5}=-(q-2)^{2}, \lambda_{S, 6}=q-4$. The coefficients are

$$
\begin{gathered}
c_{S,(1,2)}=1 \\
c_{S, j}=c^{(1)} \quad \text { for } 3 \leq j \leq 5
\end{gathered}
$$

and

$$
c_{S, 6}=c^{(2)}
$$

These coefficients have the form of Chebyshev polynomials of the second kind, as given above. There is also a zero eigenvalue $\lambda_{S, 7}=0$ with coefficient $c_{S, 7}=q\left(q^{2}-3 q+1\right)$ which does not contribute to the chromatic polynomial (4.1). The sum of the coefficients is equal to $P\left(T_{3}, q\right)=q(q-1)^{2}$ if we include $c_{S, 7}$, and is equal to $q^{2}$ if $c_{S, 7}$ is not included.

The locus $\mathcal{B}$ crosses the real $q$-axis at $q=0,2$ and $q_{c}$, where

$$
q_{c}\left(S, F B C_{y}, P B C_{x}\right)=2.702873
$$

which is the real solution of equation $2 q^{3}-11 q^{2}+24 q-24=0$. This locus separates the $q$ plane into three regions. Region $R_{1}$ extends to infinite $|q|$ and includes the intervals $q \geq q_{c}$ and $q \leq 0$ on the real $q$ axis. Region $R_{2}$ includes the real interval $2 \leq q \leq q_{c}$, while region $R_{3}$ includes the real interval $0 \leq q \leq 2$. The dominant terms in regions $R_{i}, 1 \leq i \leq 3$ are (i) $\lambda_{S, 1}$ in (4.2), (ii) $\lambda_{S, 6}$, and (iii) $\lambda_{S, 3}$ in (4.3), respectively. Therefore, $q_{c}$ for the $m \rightarrow \infty$ limit of this lattice strip $S_{m}$ is given by the degeneracy between $\left|\lambda_{S, 1}\right|$ and $\left|\lambda_{S, 6}\right|$, which yields the value in (4.7). The locus $\mathcal{B}$ and the chromatic zeros for the $L_{x}=m=20$ cyclic graph of the $S_{m}$ lattice strip are shown in Fig. 4 . This locus has support for $\operatorname{Re}(q) \geq 0$. The locus $\mathcal{B}(S)$ not only has closed regions $R_{1}$ to $R_{3}$, but also has a pair of arcs extending from $q \simeq 2.9 \pm 0.7 i$ at the boundary between $R_{1}$ and $R_{3}$ to $q \simeq 3.05 \pm 0.396 i$. 


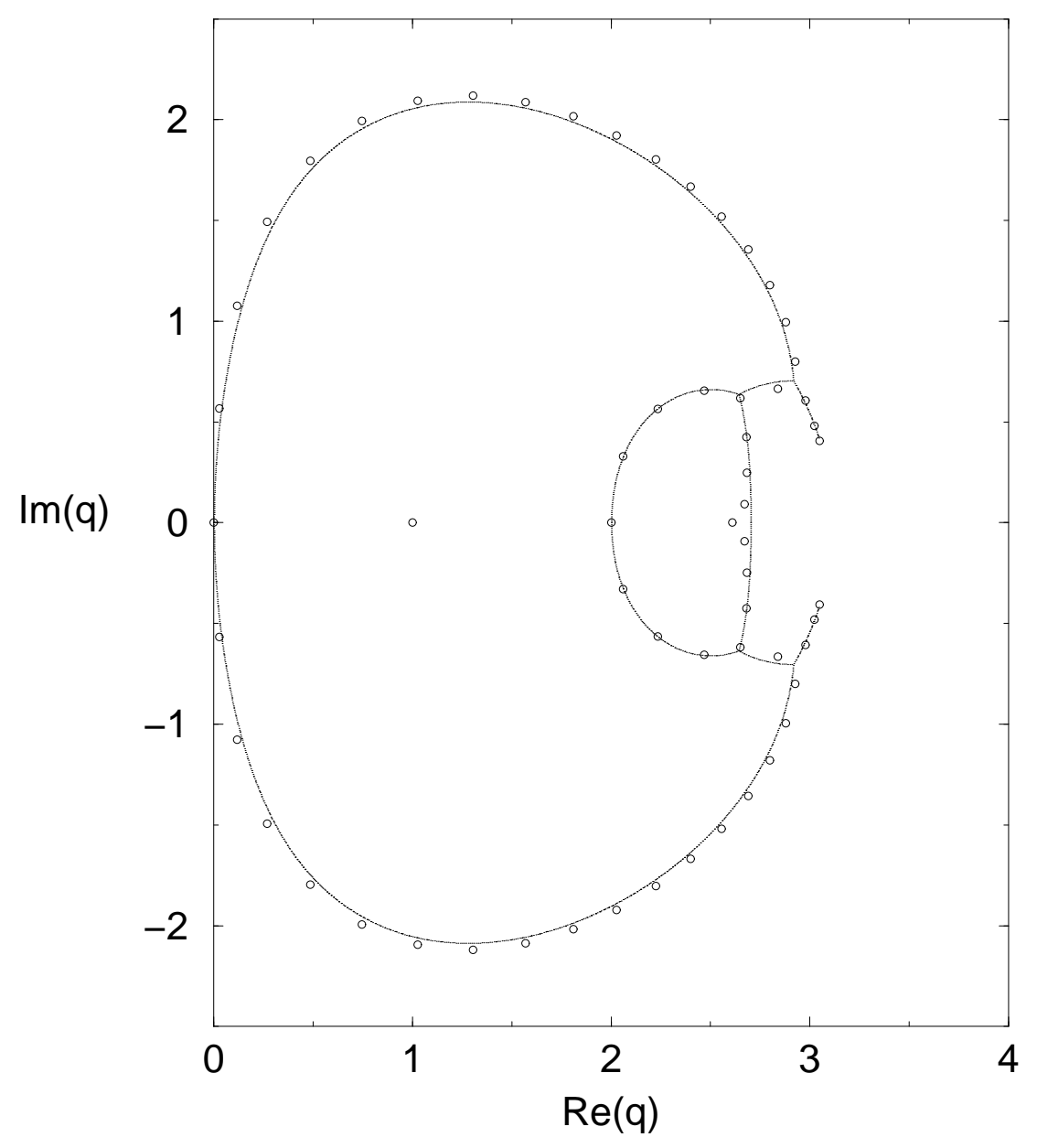

FIG. 4. Locus $\mathcal{B}$ for the $m \rightarrow \infty$ limit of the cyclic family $S_{m}$. For comparison, chromatic zeros for the graph $S_{m}$ with $m=20$ (i.e., $n=60$ ) are shown.

\section{FAMILY OF $V_{M}$ STRIPS WITH $\left(F B C_{Y}, P B C_{X}\right)$}

We next proceed to the $3 \times m$ strips of the $V_{m}$ lattice with $\left(F B C_{y}, P B C_{x}\right)$, which have one less horizontal edge in each subgraph than the $Q_{m}$ lattice, or equivalently one less diagonal edge in each subgraph than the $S_{m}$ lattice. We find $N_{V, \lambda}=6$ as for lattice $S_{m}$, and

$$
P\left(V_{m}, F B C_{y}, P B C_{x}, q\right)=\sum_{j=1}^{6} c_{V, j}\left(\lambda_{V, j}\right)^{m},
$$

where 


$$
\lambda_{V,(1,2)}=\frac{q-2}{2}\left(q^{2}-3 q+4 \pm \sqrt{(q-2)(q-3)\left(q^{2}-q+2\right)}\right),
$$

$\lambda_{V, j}$ for $j=3 \leq j \leq 5$ are roots of the cubic equation

$$
\begin{aligned}
& \xi^{3}+\left(2 q^{2}-9 q+11\right) \xi^{2} \\
& +(q-2)\left(q^{3}-8 q^{2}+20 q-14\right) \xi-(q-1)^{2}(q-2)^{2}(q-3)=0
\end{aligned}
$$

and $\lambda_{V, 6}=q-3$. The corresponding coefficients are

$$
\begin{gathered}
c_{V,(1,2)}=1 \\
c_{V, j}=c^{(1)} \text { for } 3 \leq j \leq 5
\end{gathered}
$$

and

$$
c_{V, 6}=c^{(2)} .
$$

These coefficients are the same as the coefficients for the family of lattice strips $S_{m}$, and the zero eigenvalue $\lambda_{V, 7}=0$ has the same coefficient $c_{V, 7}=q\left(q^{2}-3 q+1\right)$. Thus, the sum of the coefficients is equal to $P\left(T_{3}, q\right)=q(q-1)^{2}$ if we include $c_{V, 7}$, and is $q^{2}$ if $c_{V, 7}$ if not included.

The locus $\mathcal{B}$ has support for $\operatorname{Re}(q) \geq 0$, and consists of (1) an outer closed region which crosses the real $q$-axis at $q=0, \frac{5}{2} ;(2)$ an inner closed region which crosses the real $q$-axis at $q=2$ and $q \simeq 2.341$; and (3) a finite line segment on the the real $q$-axis extending from $q \simeq 2.341$ to $q=3$. Thus, the $q$ plane is separated into three regions and $q_{c}=3$. Region $R_{1}$ is the exterior part of the outer closed region, and extends to infinite $|q|$. Region $R_{3}$ is the interior part of the inner closed region, and region $R_{2}$ includes the interior part of the outer closed region and exterior part of $R_{3}$. The dominant terms in regions $R_{i}, 1 \leq i \leq 3$ are (i) $\lambda_{V, 1}$ in (5.2), (ii) the roots of eq. (5.3) with largest magnitude, and (iii) $\lambda_{V, 6}$, respectively. The locus $\mathcal{B}$ for the $m \rightarrow \infty$ limit of the $V_{m}$ family and the chromatic zeros for the $L_{x}=m=20$ $V_{m}$ graph are shown in Fig. 5. As was true for the $S_{m}$ lattice, The locus $\mathcal{B}(V)$ has both closed regions and finite line segment. 


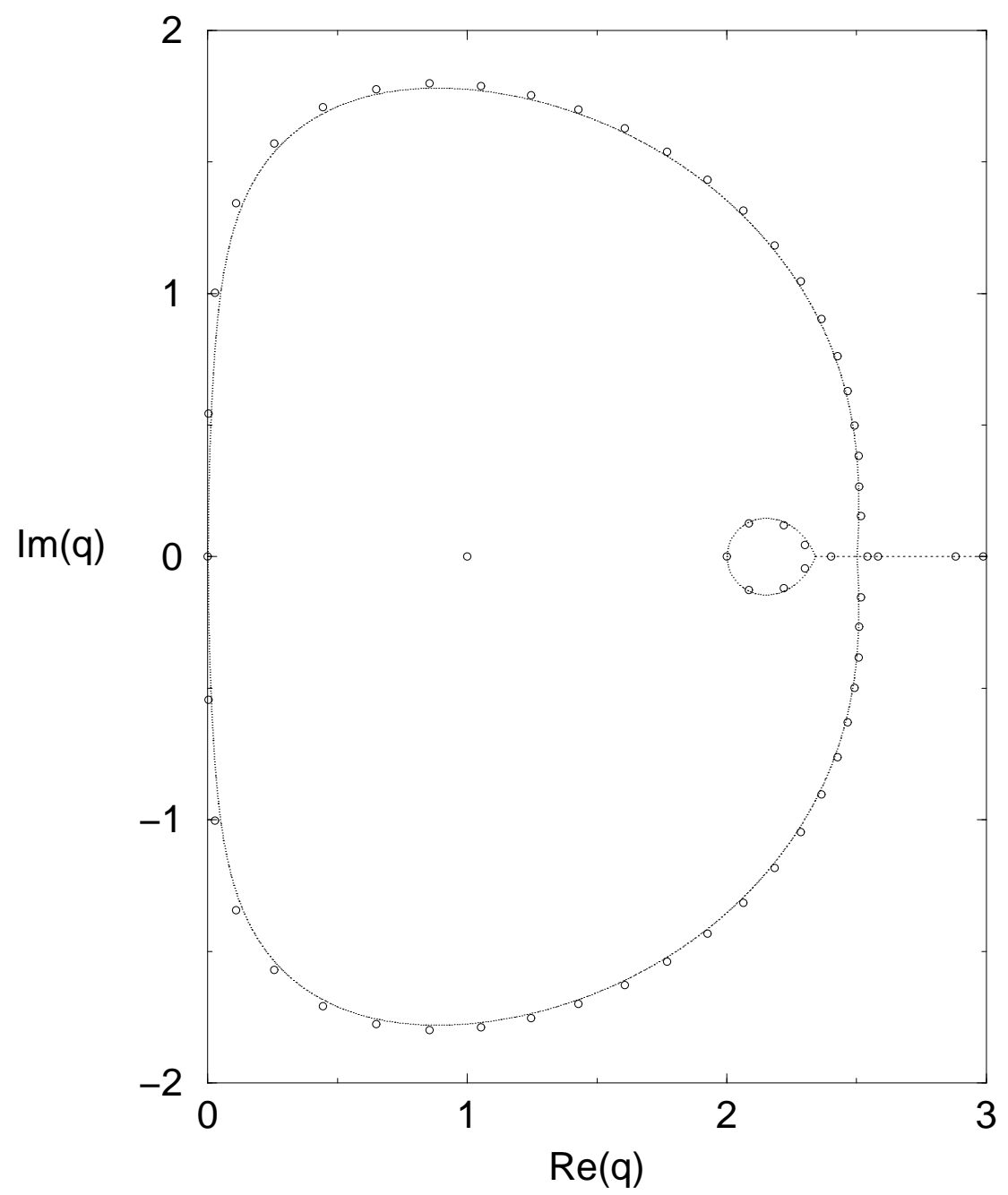

FIG. 5. Locus $\mathcal{B}$ for the $m \rightarrow \infty$ limit of the cyclic $V_{m}$ family. For comparison, chromatic zeros for the cyclic $V_{m}$ graph with $m=20$ (i.e., $n=60$ ) are shown.

\section{FAMILY OF $X_{K, M}$ STRIPS WITH $\left(F B C_{Y}, P B C_{X}\right)$}

The homeomorphic expansion of the $L_{y}=2$ square lattice with cyclic boundary condition on the horizontal edges has been studied before [27. In this section we consider the homeomorphic expansion of the $L_{y}=2$ square lattice with cyclic boundary condition on the vertical edges, so that there are $k$ vertices on the transverse direction, i.e. we make the insertion of $k-2$ degree- 2 vertices on all of the vertical edges of the strip. This is equivalent to the $L_{y}=k$ square lattice having the same boundary condition without all the internal horizontal edges. We find $N_{X, \lambda}=6$ as for lattice $V_{m}$, and 


$$
P\left(X_{k, m}, F B C_{y}, P B C_{x}, q\right)=\sum_{j=1}^{6} c_{X, j}\left(\lambda_{X_{k}, j}\right)^{m}
$$

where $\lambda_{X_{k},(1,2)}$ are the roots of the quadratic equation,

$$
\begin{aligned}
& \xi^{2}+\frac{(-1)^{k}}{q}\left(\left(q^{2}-2 q+2\right)(1-q)^{(k-1)}+q-2\right) \xi \\
& +\frac{(q-1)^{3}}{q^{2}}\left((q-1)^{(2 k-3)}-(q-2)(1-q)^{(k-2)}-1\right)=0 .
\end{aligned}
$$

The $\lambda_{X_{k},(3,4)}$ are the roots of another quadratic equation,

$$
\begin{gathered}
\xi^{2}+\frac{(-1)^{k}}{q}\left((2-q)(1-q)^{(k-1)}-2\right) \xi \\
-\frac{1}{q^{2}}\left((q-1)^{(2 k-1)}-(q-2)(1-q)^{k}-(q-1)^{2}\right)=0 \\
\lambda_{X_{k}, 5}=-(q-1) D_{k}(q)
\end{gathered}
$$

and

$$
\lambda_{X_{k}, 6}=D_{k}(q) .
$$

where $D_{k}(q)$ is defined in (1.11). In eqs. (6.2) and (6.3), the coefficient functions are polynomials for any positive integer $k$.

The corresponding coefficients are

$$
\begin{gathered}
c_{X,(1,2)}=1 \\
c_{X, j}=c^{(1)} \text { for } 3 \leq j \leq 5
\end{gathered}
$$

and

$$
c_{X, 6}=c^{(2)}
$$

These coefficients are the same as the coefficients for the lattices $S$ and $V$. Therefore, the sum of the coefficients is equal to $q^{2}$ if we do not consider the zero eigenvalue. The chromatic number is 2 and 3 when $L_{x}$ is even and odd, respectively, independent of $k$.

This lattice is the only one we consider here which does not contain a seam when we impose periodic longitudinal boundary conditions with an orientation-reversal (Möbius). We have $\lambda_{X_{k}, m b, j}=\lambda_{X_{k}, j}, 1 \leq j \leq 6$, and the corresponding coefficients are 


$$
\begin{gathered}
c_{X, m b,(1,2)}=1 \\
c_{X, m b,(3,4)}=c^{(1)} \\
c_{X, m b, 5}=-c^{(1)}
\end{gathered}
$$

and

$$
c_{X, m b, 6}=-1
$$

The changes of the coefficients when we change the longitudinal boundary condition from cyclic to Möbius follow the rules given in Theorem 6 of [9]. The chromatic number for the Möbius strips of lattice $X_{k, m}$ is 2 as $\left(k, L_{x}\right)=(o, e),(e, o)$, and 3 as $\left(k, L_{x}\right)=(o, o),(e, e)$, where $e$ and $o$ denote even and odd values for $k$ and $L_{x}$.

The fact that the $\lambda_{j}$ 's for a Möbius strip must be the same as those for the cyclic strip of the same width and lattice type was proved in [31]; this also proves, a fortiori, that (i) the total number, $N_{\lambda}$, of $\lambda_{j}$ 's, and (ii) the continuous nonanalytic locus $\mathcal{B}$, including the point $q_{c}$, are the same for the cyclic and Möbius strips. 


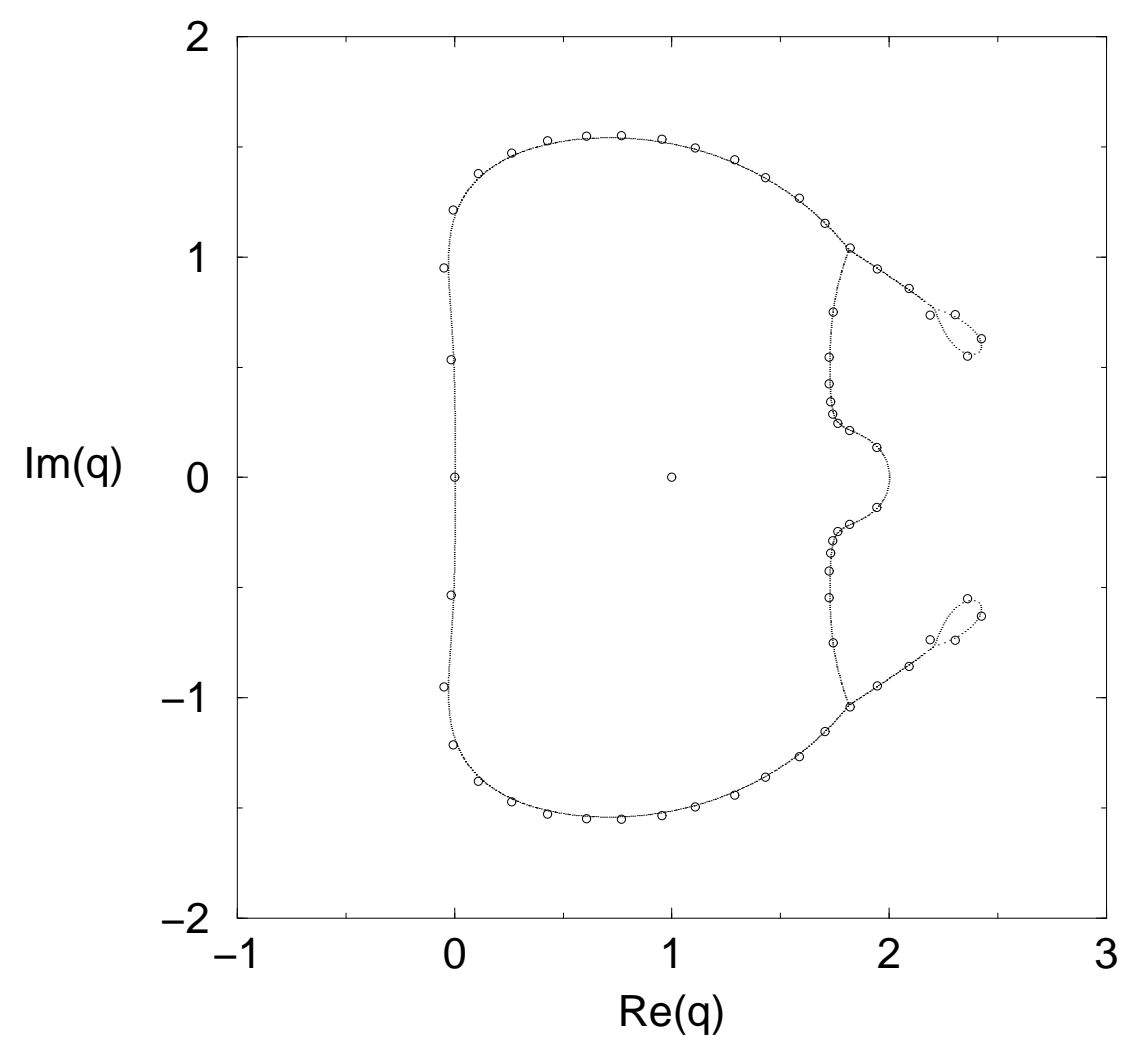

FIG. 6. Locus $\mathcal{B}$ for the $m \rightarrow \infty$ limit of the cyclic $X_{3, m}$ family and chromatic zeros for the cyclic $X_{3, m}$ graph with $m=20$ (i.e., $n=60$ ). 


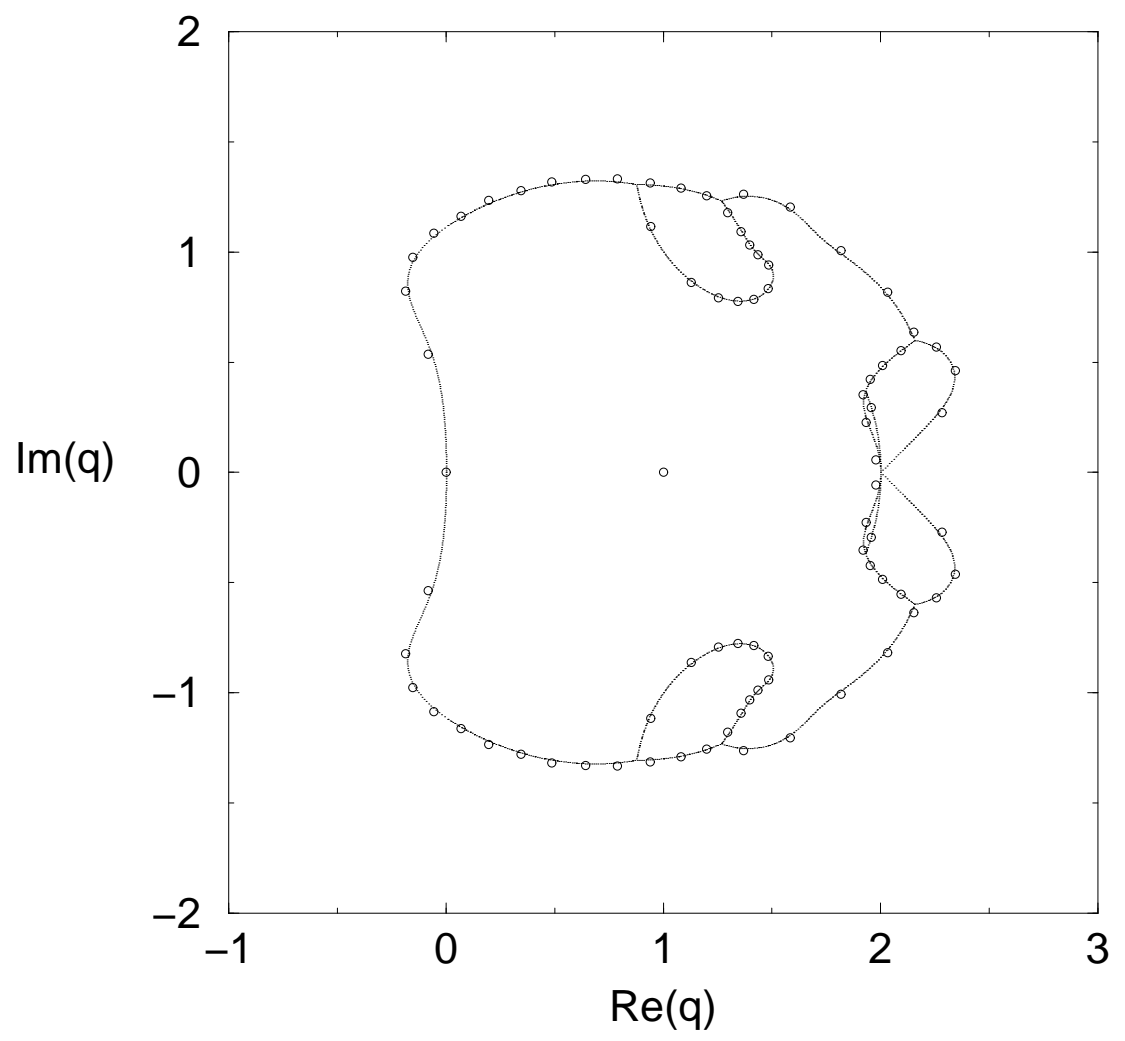

FIG. 7. Locus $\mathcal{B}$ for the $m \rightarrow \infty$ limit of the cyclic $X_{4, m}$ family and chromatic zeros for the cyclic $X_{4, m}$ graph with $m=20$ (i.e., $n=80$ ).

The locus $\mathcal{B}$ and the chromatic zeros for the $L_{x}=m=20$ cyclic graph of the $X_{k, m}$ lattice with $k=3,4$ are shown in Fig. 6 and 7 . Except for $k=2$ which is the ladder graph, we find the locus $\mathcal{B}\left(X_{k}\right)$ for $k \geq 3$ has support for $\operatorname{Re}(q) \leq 0$, and crosses the real $q$-axis at $q=0$ and 2. The two main regions are region $R_{1}$ includes the intervals $q \geq 2$ and $q \leq 0$ on the real $q$ axis, and region $R_{2}$ includes the real interval $0 \leq q \leq 2$. The dominant terms in regions $R_{1}$ and $R_{2}$ are roots of (6.2) and (6.3), respectively.

For odd $k$, there is also a pair of complex-conjugate regions $R_{3}, R_{3}^{*}$ on the upper right and lower right of $q=q_{c}=2$, which are connected to $R_{2}$ by arcs. The dominant term in these regions is $\lambda_{X_{\text {odd }}, 3}$. The locus $\mathcal{B}\left(X_{\text {odd }}\right)$ around $q_{c}$ has the form of a circular arc which bends to the left before reaching a pair of complex-conjugate roots of the discriminant of eq. (6.3). 
For even $k$, a general feature is that there are two contiguous pairs of complex-conjugate regions $R_{3}, R_{3}^{*}$ on the upper right and lower right of $q=2$ and $R_{4}, R_{4}^{*}$ on the upper left and lower left of $q=2$, and both of them touch the real $q$-axis at $q=2$. The dominant terms in $R_{3}, R_{3}^{*}$ and $R_{4}, R_{4}^{*}$ are $\lambda_{X_{\text {even }, 5}}$ and $\lambda_{X_{\text {even }, 6}, 6}$, respectively.

As $k$ increases, more small regions appear on the boundary between regions $R_{1}$ and $R_{2}$. These small regions surround part of the roots of the discriminant of eq. (6.3), as shown in Fig. 8 for $k=7$ and Fig. 9 for $k=8$. In these figures the roots of the discriminant are indicated with an asterisk, $*$. Note that the locus $\mathcal{B}$ is compact and approaches the circle $|q-1|=1$ as $k$ increases, as is illustrated in Fig. 10 for $k=15$. This is similar to what was found before for the outer envelope of the locus $\mathcal{B}$ for the homeomorphic expansions on the horizontal edges of the cyclic $L_{y}=2$ strips of the square lattices [27]. The roots of the discriminant of eq. (6.3) also approach the same circle as $k$ increases, as is illustrated in Fig. 11 for $k=50$. 


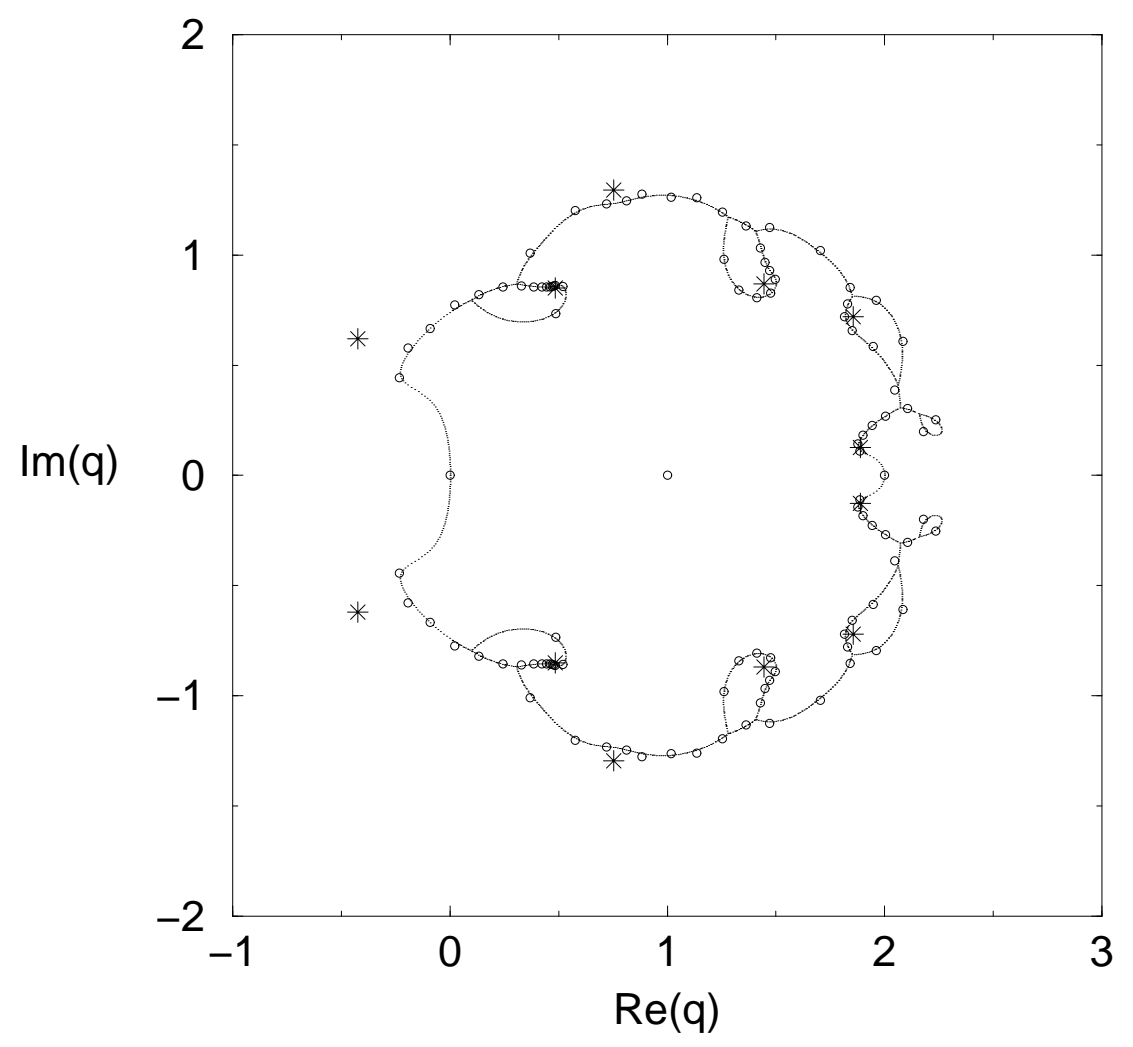

FIG. 8. Locus $\mathcal{B}$ for the $m \rightarrow \infty$ limit of the family of cyclic graphs $X_{7, m}$ and chromatic zeros for the cyclic graph $X_{7, m}$ with $m=15$ (i.e., $n=105$ ). The roots of the discriminant of eq. (6.3) are indicated with *. 


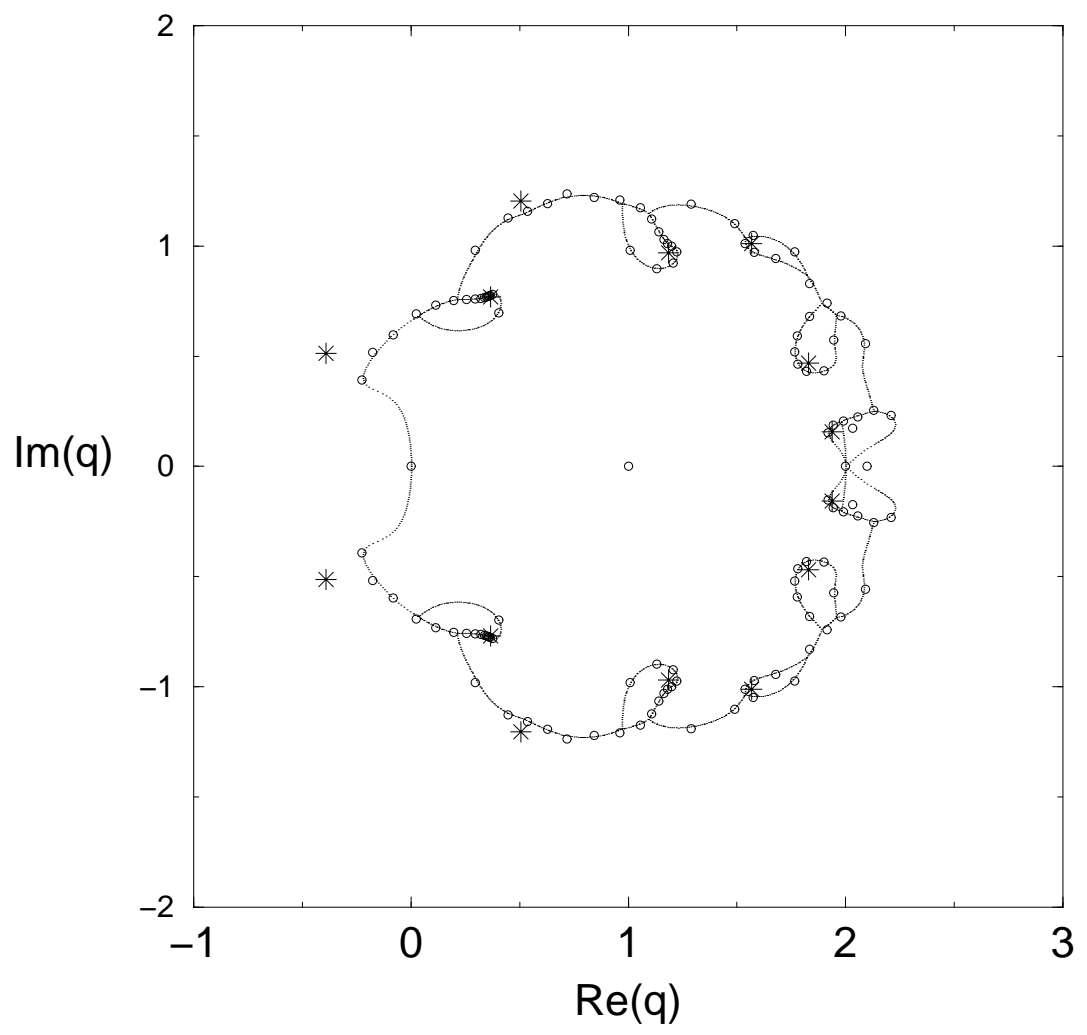

FIG. 9. Locus $\mathcal{B}$ for the $m \rightarrow \infty$ limit of the family of cyclic graphs $X_{8, m}$ and chromatic zeros for the cyclic graph $X_{8, m}$ with $m=15$ (i.e., $n=120$ ). The roots of the discriminant of eq. (6.3) are indicated with *. 


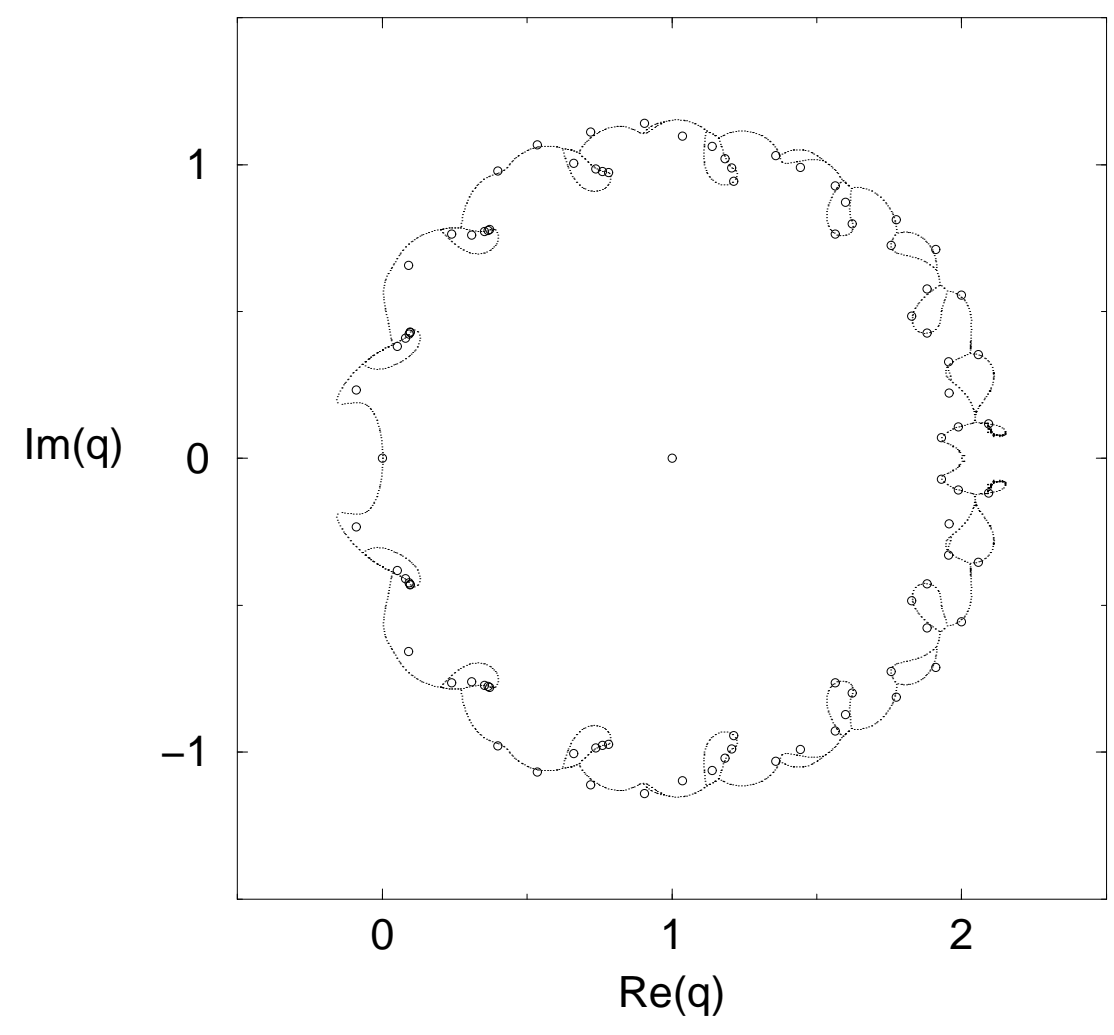

FIG. 10. Locus $\mathcal{B}$ for the $m \rightarrow \infty$ limit of the family of cyclic graphs $X_{15, m}$ and chromatic zeros for the cyclic graph $X_{15, m}$ with $m=6$ (i.e., $n=90$ ). 


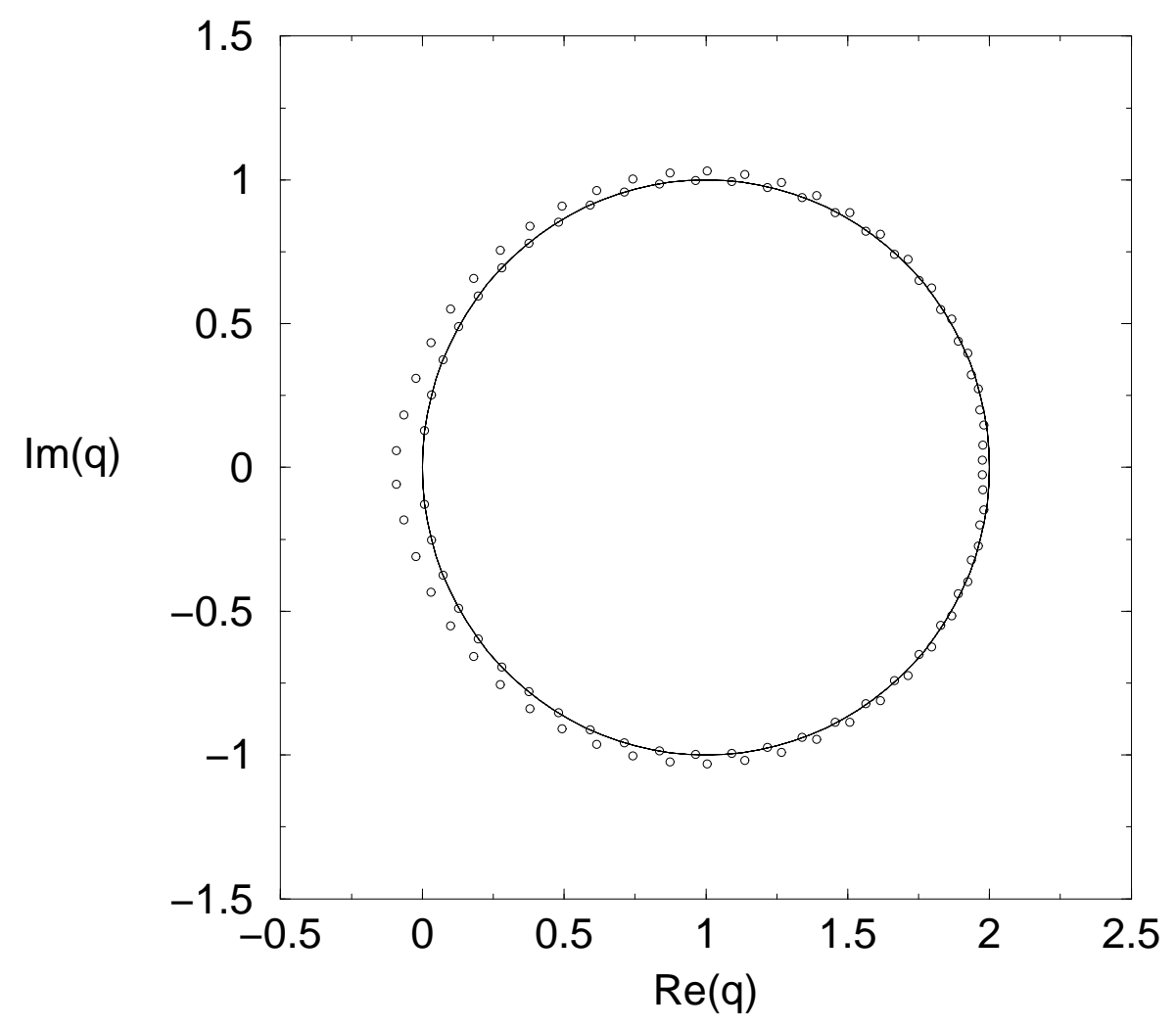

FIG. 11. The roots of the discriminant of eq. (6.3) with $k=50$. The solid circle is $|q-1|=1$.

\section{FAMILY OF $Y_{K, M}$ STRIPS WITH $\left(F B C_{Y}, P B C_{X}\right)$}

In this section we insert $k-2$ degree- 2 vertices on all the vertical edges of the $L_{y}=2$ triangular lattice with cyclic boundary conditions. Here we find $N_{Y, \lambda}=4$, and

$$
P\left(Y_{k, m}, F B C_{y}, P B C_{x}, q\right)=\sum_{j=1}^{4} c_{Y, j}\left(\lambda_{Y_{k}, j}\right)^{m}
$$

where

$$
\lambda_{Y_{k}, 1}=(q-1) D_{k+1}(q)-(q-2) D_{k}(q)
$$




$$
=D_{4}(q) D_{k}(q)+(q-1)(-1)^{(k-1)}
$$

The $\lambda_{Y_{k},(2,3)}$ are the roots of the quadratic equation,

$$
\begin{aligned}
& \xi^{2}+\frac{(-1)^{k}}{q}\left(2(2-q)(1-q)^{(k-1)}+q-4\right) \xi \\
& +\frac{1}{q^{2}}\left((q-3)(q-1)^{(2 k-1)}-(q-2)(2 q-3)(1-q)^{(k-1)}+q^{2}-3 q+3\right)=0
\end{aligned}
$$

where the coefficient functions are polynomials for positive integer $k$, and

$$
\lambda_{Y_{k}, 4}=D_{k}(q)
$$

The corresponding coefficients are

$$
\begin{gathered}
c_{Y, 1}=1 \\
c_{Y,(2,3)}=c^{(1)}
\end{gathered}
$$

and

$$
c_{Y, 4}=c^{(2)}
$$

These coefficients are the same as the coefficients for the square and triangular lattices with $L_{y}=2$ and the same boundary condition. Therefore, the sum of the coefficients is equal to $P\left(T_{2}, q\right)=q(q-1)$ if we do not consider the zero eigenvalue. The chromatic number is 2 as $\left(k, L_{x}\right)=(o, e)$, and 3 as $\left(k, L_{x}\right)=(o, o),(e, e),(e, o)$, where $e$ and $o$ denote even and odd values for $k$ and $L_{x}$. The locus $\mathcal{B}$ for the $m \rightarrow \infty$ limit of the cyclic family $Y_{k, m}$ and the chromatic zeros for the $L_{x}=m=20$ cyclic graph $Y_{k, m}$ with $k=3,4$ are shown in Fig. 12 and 13 . 


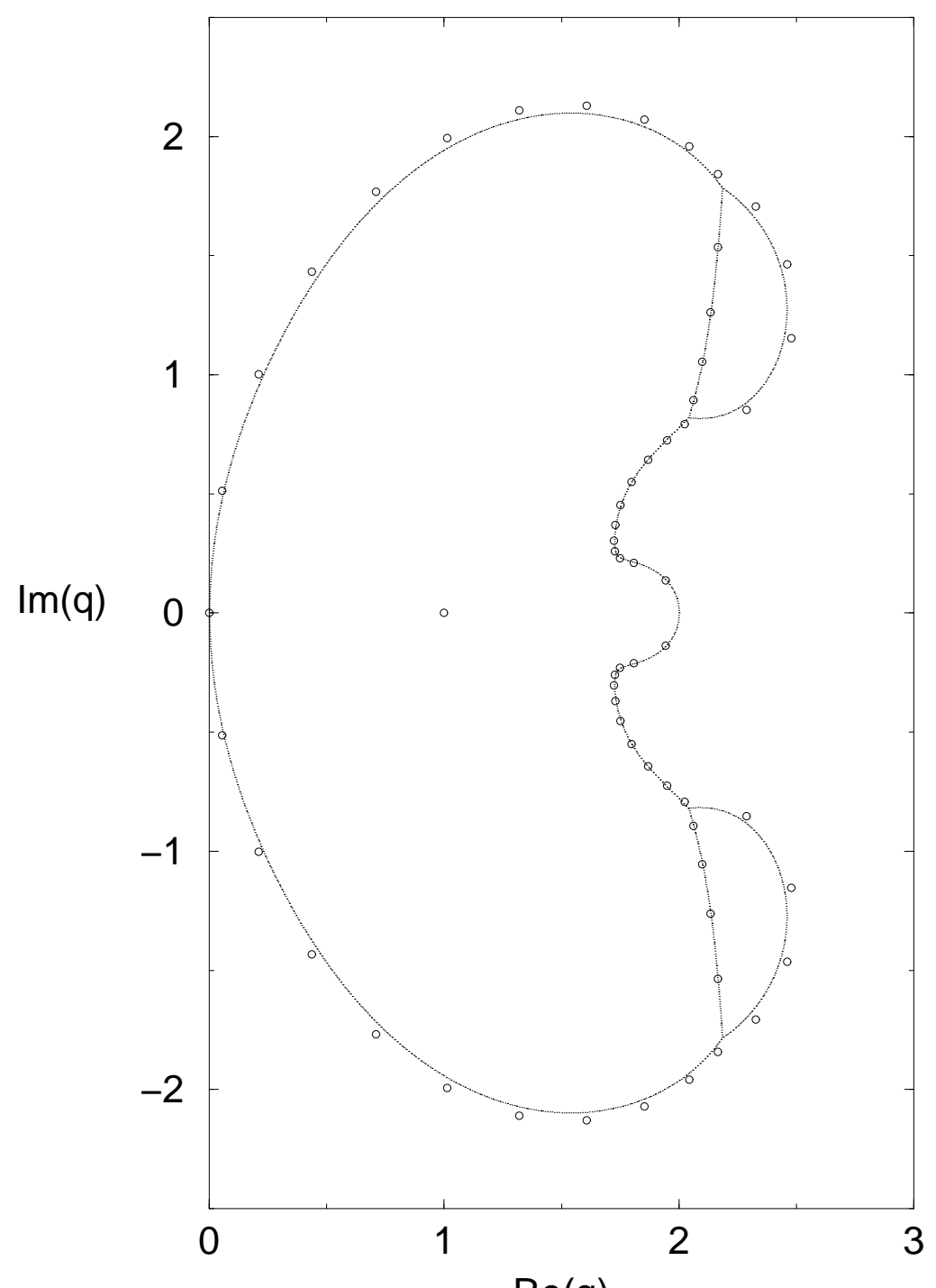

FIG. 12. Locus $\mathcal{B}$ for the $m \rightarrow \infty$ limit of the family of $\mathrm{Re}(\mathrm{g})$ (l) graphs $Y_{3, m}$ and chromatic zeros for the cyclic graph $Y_{3, m}$ with $m=20$ (i.e., $n=60$ ). 


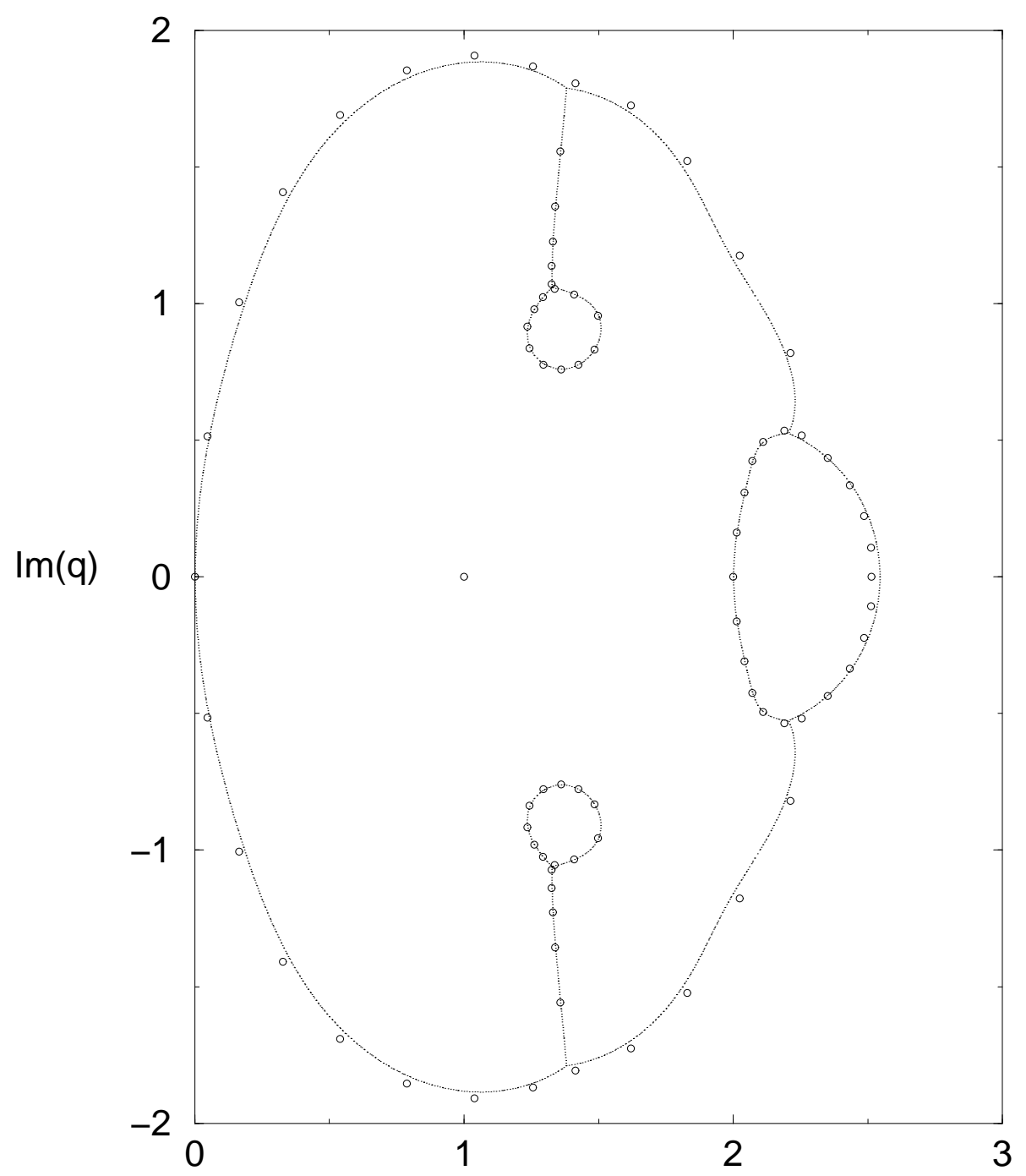

FIG. 13. Locus $\mathcal{B}$ for the $m \rightarrow \infty$ limit of the family $\operatorname{Re}(\boldsymbol{q})$ ) graphs $Y_{4, m}$ and chromatic zeros for the cyclic graph $Y_{4, m}$ with $m=20$ (i.e., $n=80$ ).

The locus $\mathcal{B}$ has support for $\operatorname{Re}(q) \geq 0$, and crosses the real $q$-axis at $q=0,2$ for odd $k$ and $q=0,2, q_{c}$ for even $k$, where $2 \leq q_{c} \leq 3$. The two main regions for odd $k$ are region $R_{1}$, which includes the intervals $q \geq 2$ and $q \leq 0$ on the real $q$ axis, and region $R_{2}$, which includes the real interval $0 \leq q \leq 2$. For even $k$, there is another region $R_{3}$ which includes the real interval $2 \leq q \leq q_{c}$, together with region $R_{1}$ including the intervals $q \geq q_{c}$ and $q \leq 0$ on the real $q$ axis. The value of this $q_{c}$ decreases as $k$ increases. The dominant terms in regions $R_{1}$ and $R_{2}$ are $\lambda_{Y, 1}$ in (7.2) and the root of eq. (7.3). For even $k$, the dominant term in $R_{3}$ is $\lambda_{Y, 4}$ in (7.4).

For $k=3$, there is also a pair of complex-conjugate regions $R_{3}, R_{3}^{*}$ contiguous to $R_{2}$, and the dominant term in these regions is $\lambda_{Y_{3}, 3}$. The $\mathcal{B}\left(Y_{k}\right)$ around $q_{c}$ again has the form of a 
circular arc that bends to the left before reaching a pair of roots of the discriminant of eq. (7.3).

For $k \geq 4$, there are more regions that appear inside $R_{2}$; in these regions, the dominant term is $\lambda_{Y_{k}, 1}$. These internal regions are connected to an arc, and the free end of the most right-hand arc is connected to the boundary between regions $R_{1}$ and $R_{2}$. The most leftland arc has two small regions on both of its ended if the small region is not connected to the boundary between regions $R_{1}$ and $R_{2}$. The free ends of these arcs are roots of the discriminant of eq. (7.3), and the small regions they are connected to also surround the roots of the discriminant. This is illustrated in Fig. 14 and Fig. 15, where the roots of the discriminant are indicated with $*$. These internal regions approach the circle $|q-1|=1$ as $k$ increases, as is indicated in Fig. 16 for $k=15$. The roots of the discriminant of eq. (7.3) also approach the same circle as $k$ increases, as is illustrated in Fig. 17 for $k=50$. 


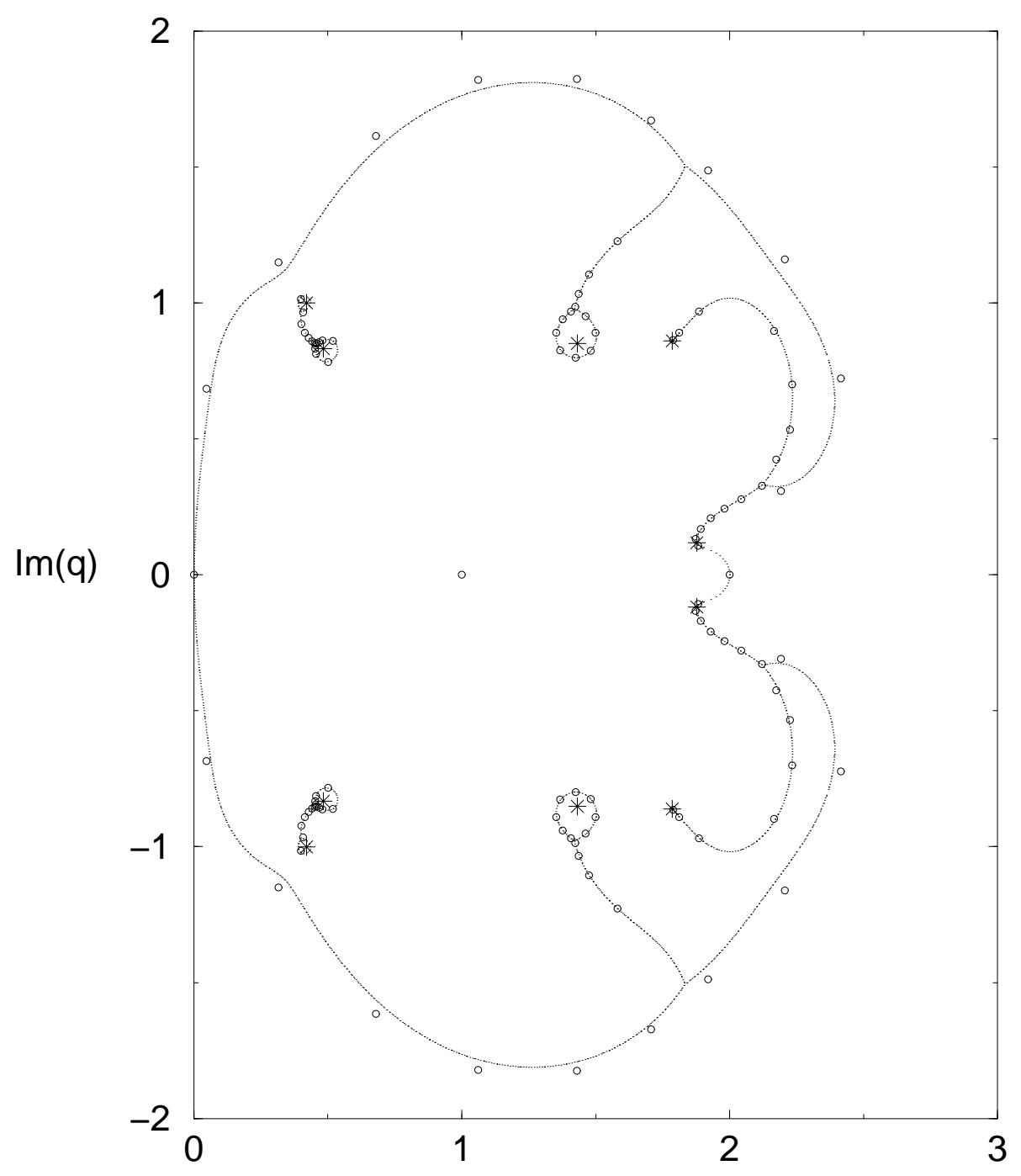

FIG. 14. Locus $\mathcal{B}$ for the $m \rightarrow \infty$ limit of the cyclic fanfie $(\mathbf{l})_{m}$ and chromatic zeros for the cyclic graph $Y_{7, m}$ with $m=15$ (i.e., $\left.n=105\right)$. The roots of the discriminant of eq. (7.3) are indicated with $*$. 


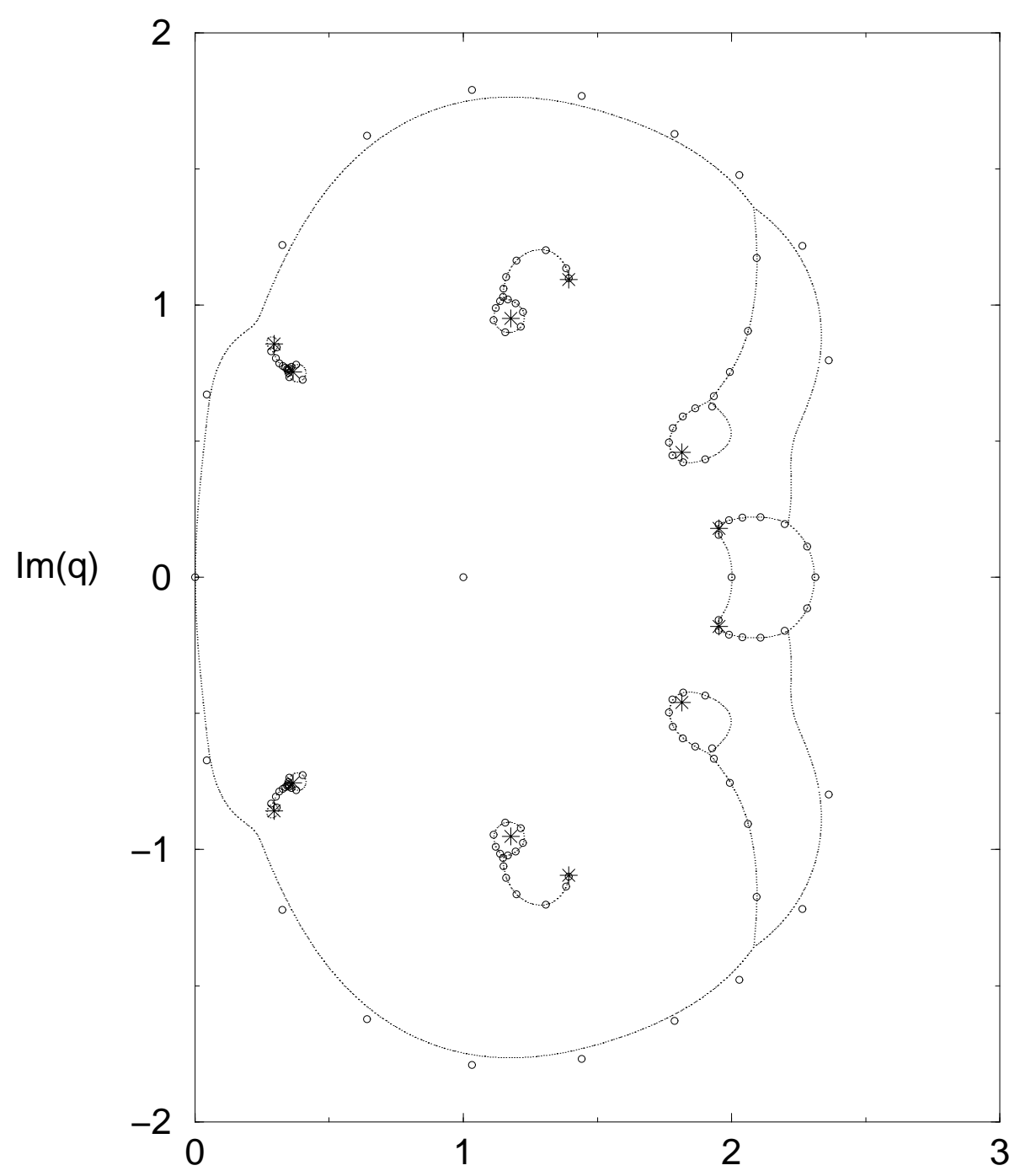

FIG. 15. Locus $\mathcal{B}$ for the $m \rightarrow \infty$ limit of the cyclic fanfie $\left(\mathbf{r}_{\mathbf{q}}\right)_{m}$ and chromatic zeros for the cyclic graph $Y_{8, m}$ with $m=15$ (i.e., $n=120$ ). The roots of the discriminant of eq. (7.3) are indicated with $*$. 


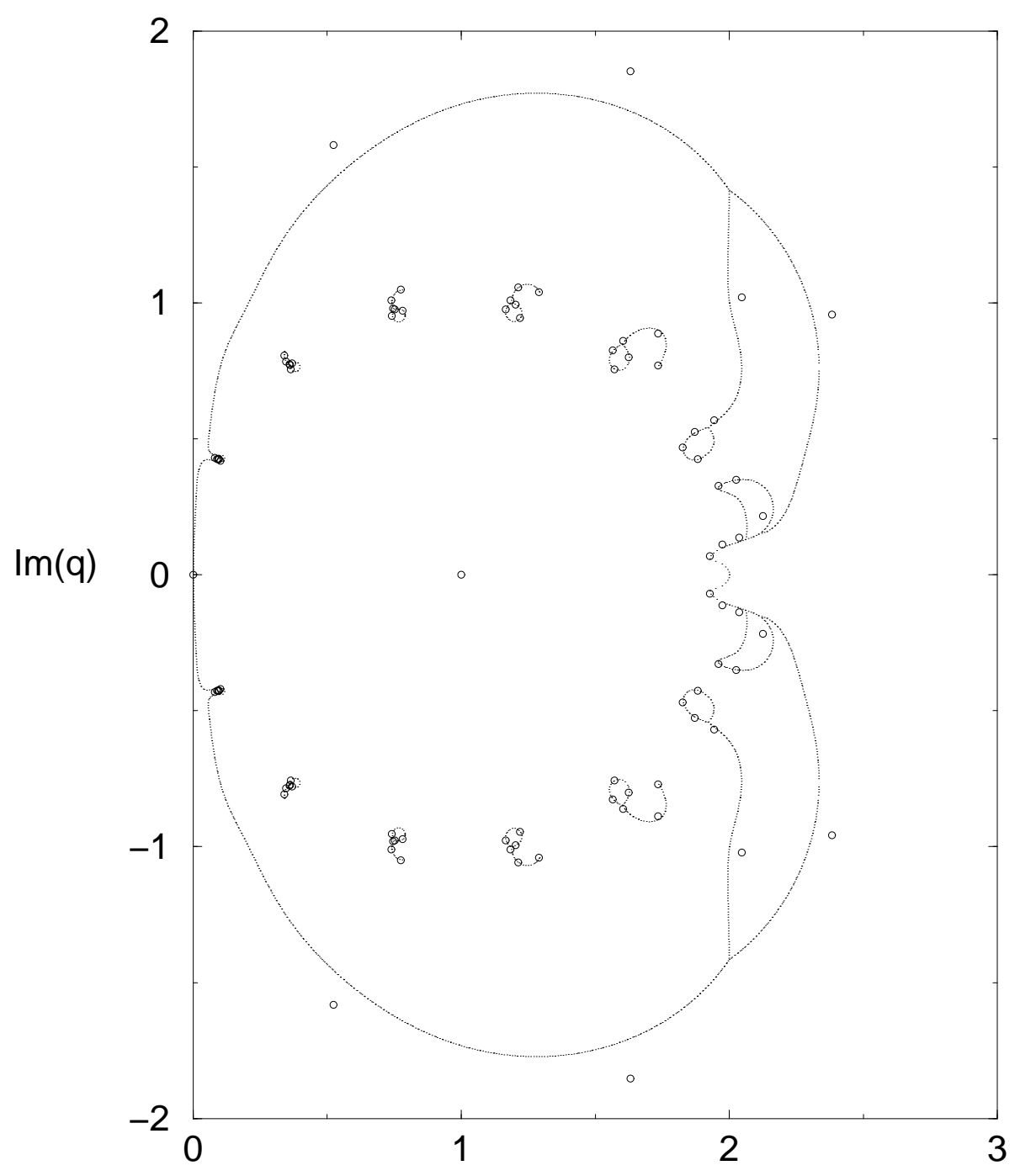

FIG. 16. Locus $\mathcal{B}$ for the $m \rightarrow \infty$ limit of the cyclic famfine $(q), m$ and chromatic zeros for the cyclic graph $Y_{15, m}$ with $m=6$ (i.e., $n=90$ ). 


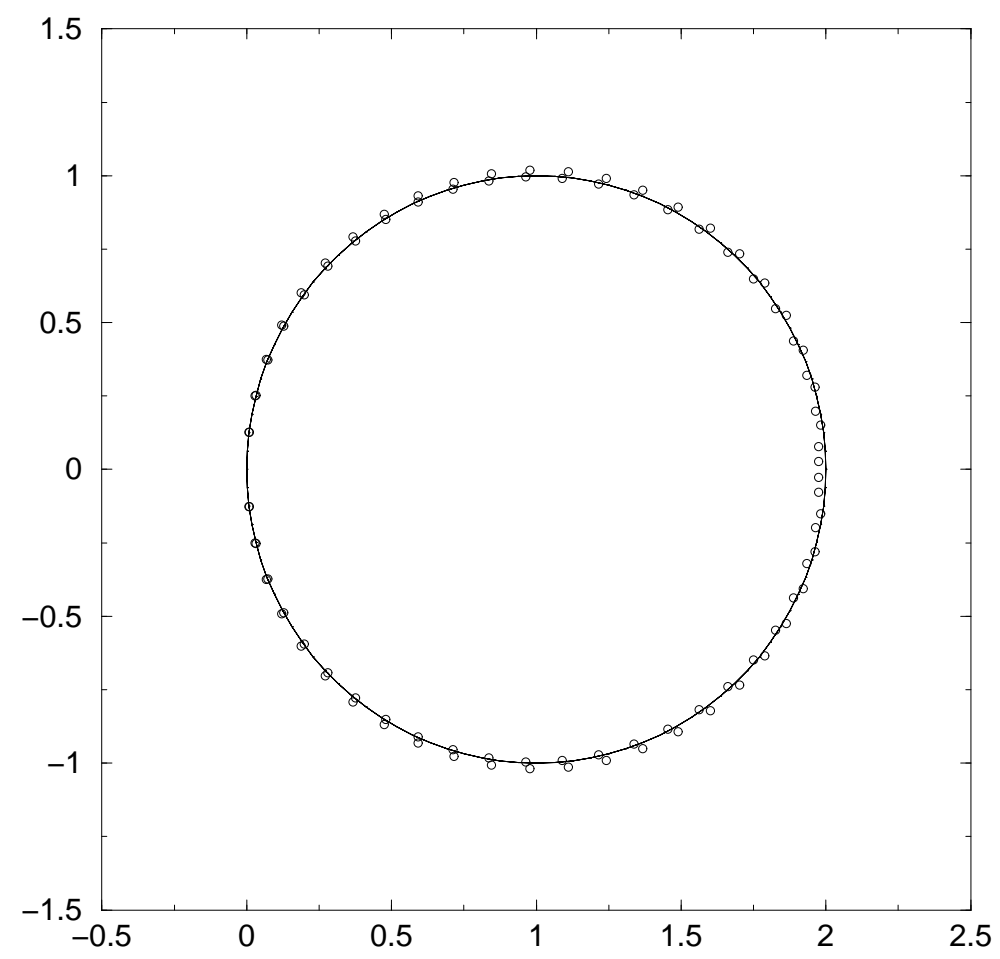

FIG. 17. The roots of the discriminant of eq. (7.3) with $k=50$. The solid circle is $|q-1|=1$.

\section{COMPARATIVE DISCUSSION}

We have found some interesting features of the locus $\mathcal{B}$ for these strips with $\left(F B C_{y}, P B C_{x}\right)=$ cyclic boundary condition:

1. For the families of the lattice strips that we have studied so far, as long as the boundary conditions are cyclic, i.e., each repeated vertex set forms a tree graph $\left(T_{L_{y}}\right)$ and the last vertex set is connected to the first vertex set to have periodic boundary conditions in the longitudinal direction, the coefficients $c_{G_{s}, j}(q)$ 's that enter into the expressions for the chromatic polynomial (1.4) are always in the form of Chebyshev polynomial 
of the second kind given in eq. (1.5). This property was found to be true for square and triangular lattices with cyclic boundary condition in [9]. Note that, while this statement applies also to certain classes of nonplanar strip graphs, such as the Möbius strips of the square lattice, it does not apply to the nonplanar strip graphs that we have analyzed in [41]; for these, we showed that the coefficients have different forms and are similar to the coefficients of the strips with doubly periodic boundary condition (torus) [7, 32, 45.

2. In general, the sum of the coefficients $C\left(G_{s}\right)$ in eq. (1.10) is the total dimension of the space of coloring configurations and should be equal to $P\left(T_{L_{y}}\right)=q(q-1)^{L_{y}-1}$ for the lattice strips with $\left(F B C_{y}, P B C_{x}\right)$ boundary conditions. Lattice $Q_{m}$ does satisfies this relation, as shown in eq. (3.9). For other lattice strips studied here, the sum of the coefficients without taking account the coefficient of the zero eigenvalue are not $P\left(T_{L_{y}}\right)$, but this can be understood easily. Consider two adjacent vertex sets of lattice $X_{k, m}$ with $k \geq 3$ and apply the coloring matrix from one vertex set to another. Effectively, only the two end vertices are connected and should be considered for coloring. Since they are separated by other vertices in between, the total number of proper colorings of these vertices is $q^{2}$. A similar argument applies to the lattice strip $V_{m}$ in Fig. 1 (d), and the lattice strip $S_{m}$ in Fig. 18 (a). For the lattice strip $O_{m}$, effectively only two connected vertices (forming a $T_{2}$ ) and a separated vertex in each vertex set are connected to another vertex set; therefore the sum of the coefficients is $q^{2}(q-1)$. With the homeomorphic expansions on the vertical edges of the cyclic $L_{y}=2$ strips of the triangular lattice, $Y_{k, m}$ does not satisfy the condition $\mathrm{C}$ given in 43. However, it is equivalent to the homeomorphic expansions on the diagonal edges of the same lattice 
strip, as shown in Fig. 18 (b), so the sum of the coefficients is still $q(q-1)$.
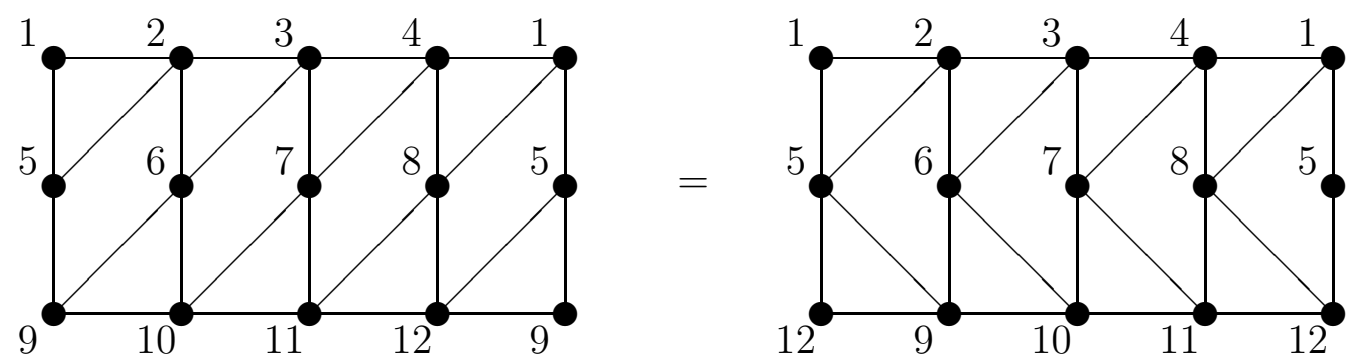

(a)
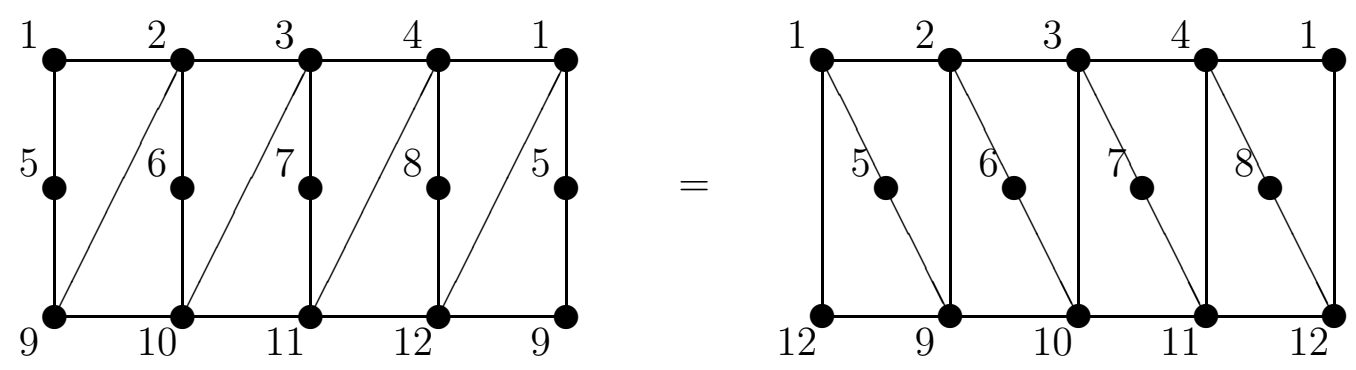

(b)

FIG. 18. Illustrative deformation for the strip graphs of (a) $S_{m}$ lattice with $L_{y}=3$ and (b) $Y_{k, m}$ lattice with $k=3$, length $L_{x}=m=4$ and $\left(F B C_{y}, P B C_{x}\right)=$ cyclic boundary conditions.

3. The loci $\mathcal{B}$ 's for the infinite-length limits of the strips studied here with global circuits in the longitudinal direction cross the real $q$-axis at $q=0,2$ as before. The feature of the cyclic strips that there is a correlation between the coefficient $c_{G_{s}, j}$ of the respective dominant $\lambda_{G_{s}, j}$ 's in regions that include intervals of the real axis discussed in [7] is found to be true again. The $c_{G_{s}, j}$ 's of the dominant $\lambda_{G_{s}, j}$ 's in (1) region $R_{1}$ containing the intervals $q \geq q_{c}$ and $q \leq 0$, (2) region containing the intervals $0<q<2$, and (3) region containing the interval $2<q<q_{s}$, where $q_{s}$ may not be $q_{c}$, are $c^{(0)}=1, c^{(1)}$, and $c^{(2)}$, respectively.

4. In previous calculations of $\mathcal{B}$ for the zero-temperature Potts antiferromagnet on infinitelength limits of strip graphs with global circuits (i.e., $P B C_{x}$ ) [6, 30, 27, 28, 31, 32, 40, (7), the respective loci did not involve arc endpoints. Here we find such arcs with endpoints such that one end is connected to the boundary of regions for the infinite-length limits of the families $S, V$, and $Y_{k}$ with $k \geq 7$, and arcs connecting two regions for lattices $V$, $X_{\text {odd }}$ and $Y_{k}$ with $k \geq 4$. In Table \we list various properties of these lattices presented 
in this work.

5. Although some loci $\mathcal{B}$ calculated for the infinite-length limits of families of graphs with global circuits involved disconnected pieces (e.g., [26,27]), here we find a series of such disconnected components, with a remarkably intricate structure, as is evident in Figs. 14, 15, and 16.

TABLE I. Properties of $P, W$, and $\mathcal{B}$ for strip graphs $G_{s}$ of lattices studied here. The properties apply for a given strip of type $G_{s}$ of size $L_{y} \times L_{x}$; some apply for arbitrary $L_{x}$, such as $N_{\lambda}$, while others apply for the infinite-length limit, such as the properties of the locus $\mathcal{B}$. The boundary conditions is $\left(F B C_{y}, P B C_{x}\right)$ $=$ cyclic. The column denoted eqs. describes the numbers and degrees of the algebraic equations giving the $\lambda_{G_{s}, j}$; for example, $\{2(1), 2(2), 1(4)\}$ indicates that there are 2 linear equations, 2 quadratic equations and one quartic equation. The column denoted BCR lists the points at which $\mathcal{B}$ crosses the real $q$ axis; the largest of these is $q_{c}$ for the given family $G_{s}$. The notation "int; $q_{1} ; q_{c}$ " refers to cases where $\mathcal{B}$ contains a real interval, there is a crossing at $q_{1}$, and the right-hand endpoint of the interval is $q_{c}$. $q_{c}$ for lattice $Y_{k, m}$ with even $k$ depends on the value of $k$, and is not shown here. Column labeled "SN" refers to whether $\mathcal{B}$ has $\underline{\text { support for }}$ negative $\operatorname{Re}(q)$, indicated as yes (y) or no (n).

\begin{tabular}{|c|r|r|c|c|c|}
\hline \hline \hline$G_{s}$ & $L_{y}$ & $N_{\lambda}$ & eqs. & BCR & SN \\
\hline \hline $\mathrm{O}$ & 4 & 14 & $\{1(1) 1(3) 1(4) 1(6)\}$ & $0,2,2.638$ & $\mathrm{y}$ \\
\hline $\mathrm{Q}$ & 3 & 10 & $\{2(1) 2(2) 1(4)\}$ & $0,2,3$ & $\mathrm{n}$ \\
\hline $\mathrm{S}$ & 3 & 6 & $\{2(1) 2(2)\}$ & $0,2,2.703$ & $\mathrm{n}$ \\
\hline $\mathrm{V}$ & 3 & 6 & $\{1(1) 1(2) 1(3)\}$ & $0,2,2.341$, int: 2.5; 3 & $\mathrm{n}$ \\
\hline $\mathrm{X}$ & $\mathrm{k}$ & 6 & $\{2(1) 2(2)\}$ & 0,2 & $\mathrm{y}$ \\
\hline $\mathrm{Y}$ & $\mathrm{k}$ & 4 & $\{2(1) 1(2)\}$ & $0,2, q_{c}\left(k_{\text {even }}\right)$ & $\mathrm{n}$ \\
\hline \hline \hline
\end{tabular}

\section{CONCLUSIONS}

In this paper we have presented exact calculations of the zero-temperature $q$-state Potts antiferromagnet partition function (equivalently, chromatic polynomial $P$ ), for various lattice strips of fixed width $L_{y}$ and arbitrarily great length $L_{x}$ with cyclic boundary conditions $\left(F B C_{y}, P B C_{x}\right)$. The coefficient $c_{G, j}$ of degree $d$ in $q$ is $c^{(d)}=U_{2 d}\left(\frac{\sqrt{q}}{2}\right)$, where $U_{n}(x)$ is the Chebyshev polynomial of the second kind. We explain the sums of the coefficients for the respective families. We also find a number of interesting and novel features of the singular locus $\mathcal{B}$. The results herein are thus of interest both from the viewpoint of exact results in statistical mechanics, graph theory, and algebraic geometry. 


\section{APPENDIX: FAMILY OF $Z_{M}$ STRIPS WITH $\left(P B C_{Y}, P B C_{X}\right)$}

In this section we consider the $4 \times m$ strips of the square lattice with $\left(P B C_{y}, P B C_{x}\right)$ boundary conditions (torus), but with two diagonal edges in each basic vertex set, i.e., instead of $L_{x}$ set of tree graphs, we have $L_{x}$ set of complete graphP $K_{4}$ (tetrahedron) to begin with and $e=\{11,22,33,44\}$. The lattice with length $L_{x}=m$ will be denoted as $Z_{m}$, and an illustrative example with $m=4$ is displayed in Fig. 19 .

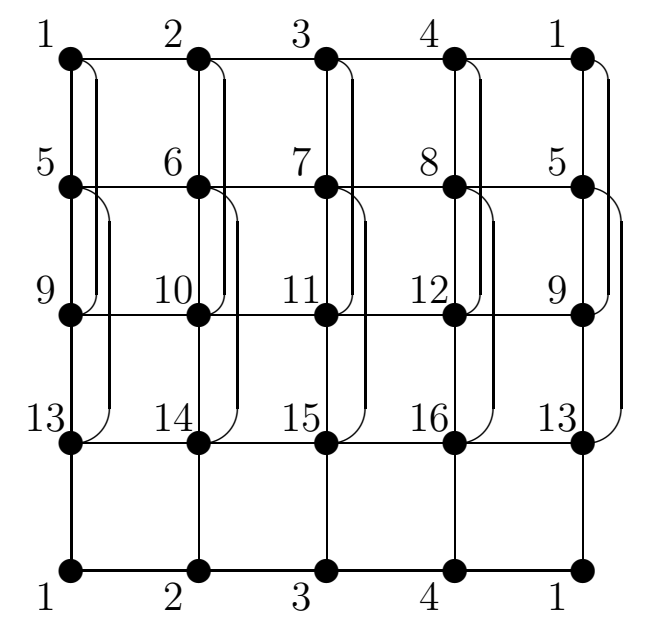

FIG. 19. Illustrative strip graphs of $Z_{m}$ lattice with $L_{y}=4$ with length $L_{x}=m=4$ and $\left(P B C_{y}, P B C_{x}\right)$ $=$ torus boundary condition. Vertices are indicated with $\bullet$ (points where edges cross without a symbol $\bullet$ are not vertices.)

In [43], the lattice with $L_{x}$ set of $K_{L_{y}}$ for arbitrary $L_{y}$ is called the bracelet strip, and the $\lambda$ 's with coefficients $1, O(q)$, and $O\left(q^{2}\right)$ are given. For $L_{y}=2$, it is the ladder graph with vertex set $T_{2}=K_{2}$. For lattice strip with $L_{y}=3$, which has vertex set $C_{3}=K_{3}$, the chromatic polynomial was given in [32]. Here we give the full set of $\lambda$ 's for the $L_{y}=4$ strip. We find $N_{Z, \lambda}=16$ and

$$
P\left(Z_{m}, P B C_{y}, P B C_{x}, q\right)=\sum_{j=1}^{16} c_{Z, j}\left(\lambda_{Z, j}\right)^{m},
$$

where all $\lambda_{Z, j}$ 's for $1 \leq j \leq 16$ are polynomials:

\footnotetext{
${ }^{2} K_{n}$ denotes the complete graph, i.e. the graph with $n$ vertices such that each vertex is connected by an edge to all of the other vertices.
} 


$$
\begin{gathered}
\lambda_{Z, 1}=q^{4}-10 q^{3}+41 q^{2}-84 q+73 \\
\lambda_{Z, 2}=-\left(q^{3}-12 q^{2}+50 q-73\right) \\
\lambda_{Z, 3}=-(q-3)\left(q^{2}-5 q+7\right) \\
\lambda_{Z, 4}=q^{2}-9 q+21 \\
\lambda_{Z, 5}=q^{2}-5 q+5 \\
\lambda_{Z, 6}=q^{2}-11 q+31 \\
\lambda_{Z, 7}=q^{2}-5 q+7 \\
\lambda_{Z, 13}=5-q \\
\lambda_{Z, 1}=q^{2}-7 q+11 \\
\lambda_{Z, 10}=2-q \\
\end{gathered}
$$

and $\lambda_{Z, 16}=1$. The corresponding coefficients are

$$
c_{Z, 1}=1
$$




$$
\begin{gathered}
c_{Z, 2}=\frac{1}{3} c_{Z, 3}=q-1 \\
c_{Z, 4}=c_{Z, 5}=\frac{3}{2}(q-1)(q-2) \\
c_{Z, 6}=\frac{1}{2} c_{Z, 7}=\frac{1}{3} c_{Z, 8}=\frac{1}{2} q(q-3) \\
c_{Z, 9}=\frac{1}{3} c_{Z, 13}=\frac{1}{6}(q-1)(q-2)(q-3) \\
c_{Z, 10}=c_{Z, 14}=\frac{3}{2} c_{Z, 12}=q(q-2)(q-4) \\
c_{Z, 15}=\frac{1}{3} c_{Z, 11}=\frac{1}{6} q(q-1)(q-5)
\end{gathered}
$$

and

$$
c_{Z, 16}=q^{4}-10 q^{3}+29 q^{2}-24 q+1
$$

The sum of all of the coefficients is equal to $P\left(C_{4}, q\right)=q(q-1) D_{4}(q)$. The chromatic number is $\chi(Z)=4$.

The locus $\mathcal{B}$ and chromatic zeros for the $L_{x}=m=20$ cyclic graph of the $Z_{m}$ lattice are shown in Fig. 20. The locus $\mathcal{B}$ crosses the real $q$-axis at $q=0,2$ and $q_{c}$, where

$$
q_{c}\left(Z, P B C_{y}, P B C_{x}\right) \simeq 3.673593
$$




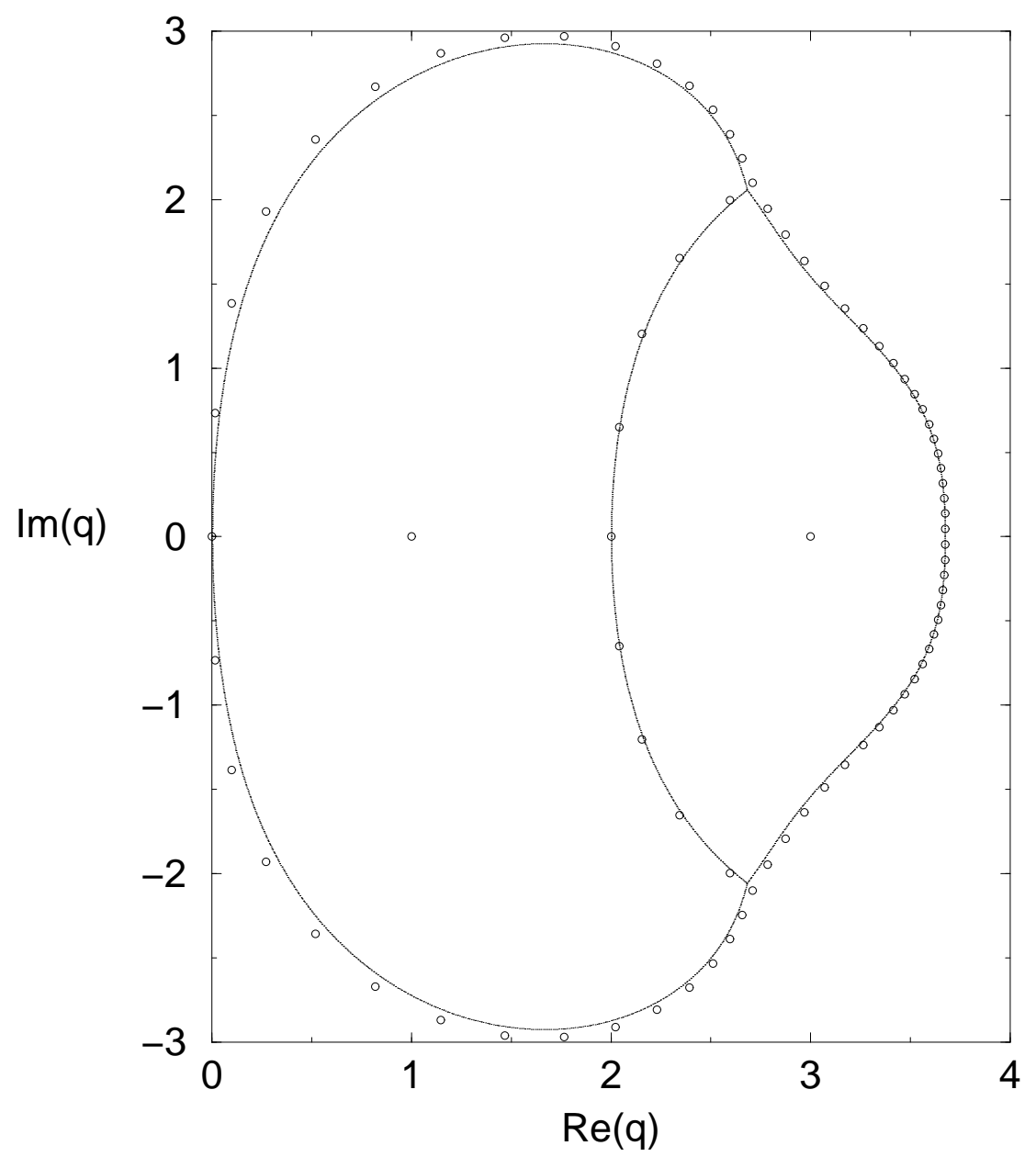

FIG. 20. Locus $\mathcal{B}$ for the $m \rightarrow \infty$ limit of the family $Z_{m}$ with toroidal boundary conditions and chromatic zeros for $Z_{m}$ with $m=20$ (i.e., $n=80$ ).

The locus $\mathcal{B}$ has support for $\operatorname{Re}(q) \geq 0$, and separates the $q$ plane into three regions. The outermost one, region $R_{1}$, extends to infinite $|q|$ and includes the intervals $q \geq q_{c}$ and $q \leq 0$ on the real $q$ axis. Region $R_{2}$ includes the real interval $2 \leq q \leq q_{c}$, while region $R_{3}$ includes the real interval $0 \leq q \leq 2$. In regions $R_{i}, 1 \leq i \leq 3$, the dominant terms are $\lambda_{Z, 1}, \lambda_{Z, 6}$, and $\lambda_{Z, 2}$, respectively. Thus, the $q_{c}(Z)$ given in (10.25) is the degeneracy between $\left|\lambda_{Z, 1}\right|$ and $\left|\lambda_{Z, 6}\right|$, and is the real solution of $q^{3}-9 q^{2}+31 q-42$.

The locus $\mathcal{B}$ has three regions and crosses the real $q$-axis at $q=0,2$, and $q_{c}$, which is quite similar to the $\mathcal{B}$ for the same lattice strip with $L_{y}=3$ [32] but has a larger size. Since the dominant $\lambda$ 's in these three regions have coefficients with order $O(1), O(q)$, and $O\left(q^{2}\right)$, which are called level 0,1 , and 2 in [43], naively one may infer this to be true for arbitrary 
$L_{y}$. The locus $\mathcal{B}$ determined from the eight general $\lambda$ 's given in 43 for $3 \leq L_{y} \leq 10$ is shown in Fig. 21, where the envelope of the outer boundary of $\mathcal{B}$ does increase in size as $L_{y}$ increases. However, for $L_{y}=8,9$, and 10, an extra small regions $R_{4}$ appears around $q=7,8$, and 8 , respectively. Therefore, even for such simple structure of $\lambda$ 's (in the form of polynomial), the locus $\mathcal{B}$ may not remain simple for sufficiently large $L_{y}$.

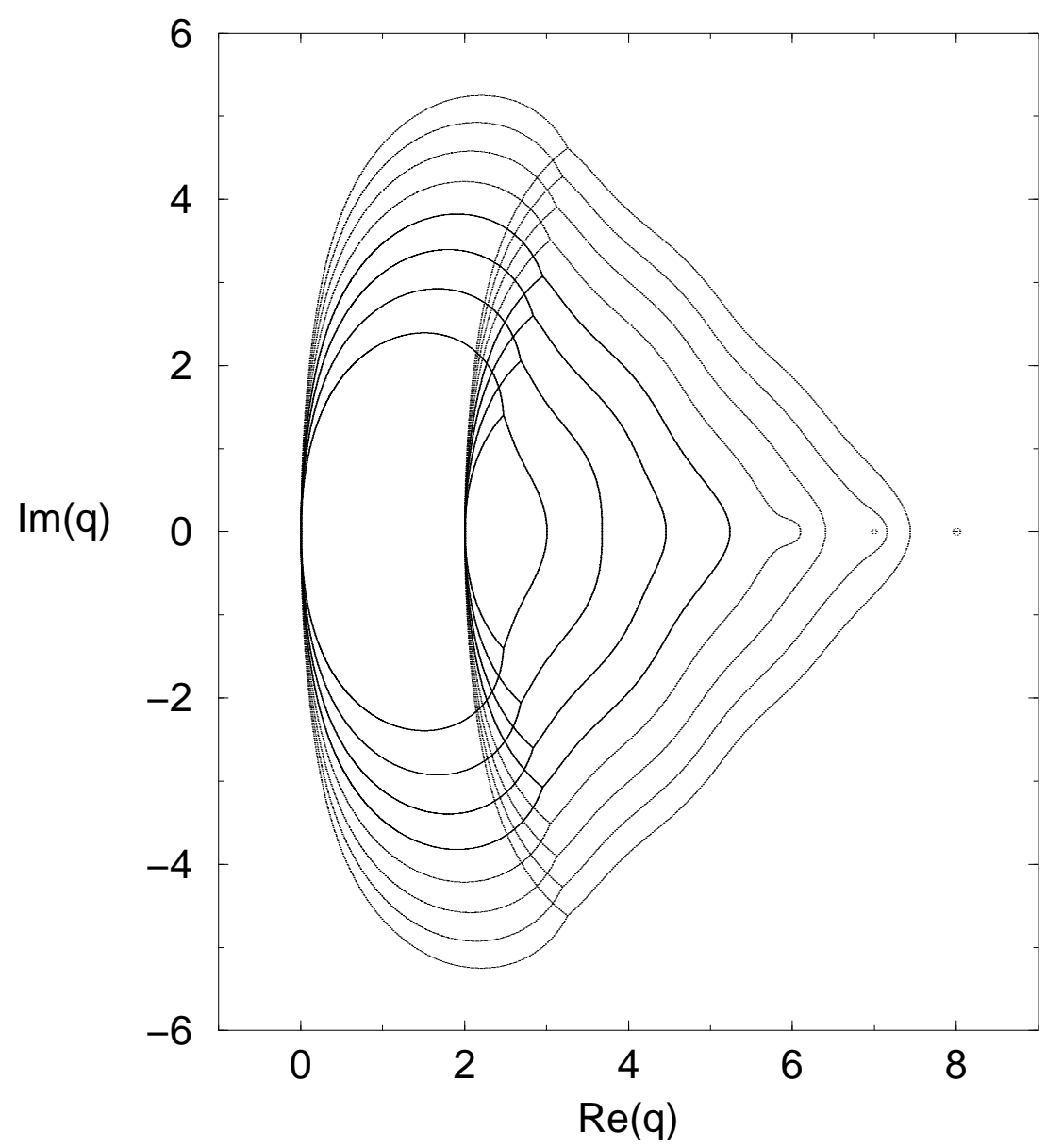

FIG. 21. Loci $\mathcal{B}$ 's for the infinite-length limit of the square lattice strip with $K_{L_{y}}$ as the basic subgraph vertex set and $\left(P B C_{x}, P B C_{y}\right)=$ torus boundary conditions. In order of the sizes of the main envelopes from small to large, the $\mathcal{B}$ 's are for $3 \leq L_{y} \leq 10$.

Acknowledgment: I would like to thank Prof. R. Shrock for helpful discussions. 
[1] R. B. Potts, Proc. Camb. Phil. Soc. 48 (1952) 106.

[2] F. Y. Wu, Rev. Mod. Phys. 54 (1982) 235.

[3] R. C. Read, J. Combin. Theory 4 (1968) 52.

[4] R. C. Read and W. T. Tutte, "Chromatic Polynomials", in Selected Topics in Graph Theory, 3, eds. L. W. Beineke and R. J. Wilson (Academic Press, New York, 1988.).

[5] N. L. Biggs, Algebraic Graph Theory (2nd ed., Cambridge Univ. Press, Cambridge, 1993).

[6] R. Shrock and S.-H. Tsai, Phys. Rev. E55 (1997) 5165.

[7] S.-C. Chang and R. Shrock, cond-mat/0005236.

[8] S. Beraha, J. Kahane, and N. Weiss, J. Combin. Theory B 27 (1979) 1; ibid., 28 (1980) 52.

[9] S.-C. Chang and R. Shrock, Physica A, in press (cond-mat/0005232).

[10] E. H. Lieb, Phys. Rev. 162 (1967) 162.

[11] N. L. Biggs, R. M. Damerell, and D. A. Sands, J. Combin. Theory B 12 (1972) 123.

[12] N. L. Biggs and G. H. Meredith, J. Combin. Theory B20 (1976) 5.

[13] N. L. Biggs, Bull. London Math. Soc. 9 (1977) 54.

[14] R. C. Read, in Proc. 3rd Caribbean Conf. on Combin. and Computing (1981).

[15] R. C. Read, in Proc. 5th Caribbean Conf. on Combin. and Computing (1988).

[16] R. J. Baxter, J. Phys. A 20 (1987) 5241.

[17] R. C. Read and G. F. Royle, in Graph Theory, Combinatorics, and Applications (Wiley, NY, 1991), vol. 2, p. 1009.

[18] R. Shrock and S.-H. Tsai, Phys. Rev. E55 (1997) 6791.

[19] R. Shrock and S.-H. Tsai, Phys. Rev. E56 (1997) 1342, 2733, 4111.

[20] R. Shrock and S.-H. Tsai, Phys. Rev. E56 (1997) 3935.

[21] J. Salas and A. Sokal, J. Stat. Phys. 86 (1997) 551.

[22] M. Roček, R. Shrock, and S.-H. Tsai, Physica A252 (1998) 505.

[23] M. Roček, R. Shrock, and S.-H. Tsai, Physica A259 (1998) 367.

[24] R. Shrock and S.-H. Tsai, Physica A259 (1998) 315.

[25] R. Shrock and S.-H. Tsai, Phys. Rev. E58 (1998) 4332; cond-mat/9808057.

[26] R. Shrock and S.-H. Tsai, J. Phys. A 31 (1998) 9641; Physica A265 (1999) 186.

[27] R. Shrock and S.-H. Tsai, J. Phys. A Lett. 32 (1999) L195.

[28] R. Shrock and S.-H. Tsai, J. Phys. A 32 (1999) 5053.

[29] R. Shrock and S.-H. Tsai, Phys. Rev. E60 (1999) 3512.

[30] R. Shrock and S.-H. Tsai, Physica A 275 (2000) 429.

[31] R. Shrock, Phys. Lett. A261 (1999) 57.

[32] N. L. Biggs and R. Shrock, J. Phys. A (Letts) 32, L489 (1999).

[33] A. Sokal, cond-mat/9904146.

[34] R. Shrock, in the Proceedings of the 1999 British Combinatorial Conference, BCC99 (July, 1999), Discrete Math., in press.

[35] R. Shrock, Physica A281, 221 (2000). 
[36] N. L. Biggs, reports LSE-CDAM-99-03,05.

[37] S.-C. Chang and R. Shrock, Phys. Rev. E62, 4650 (2000).

[38] R. Shrock, Physica A283, 388 (2000).

[39] S.-C. Chang and R. Shrock, Physica A286, 189 (2000).

[40] S.-C. Chang and R. Shrock, Physica A, in press (cond-mat/0004161).

[41] S.-C. Chang and R. Shrock, Phys. Rev. E62, 4650 (2000).

[42] S.-C. Chang and R. Shrock, cond-mat/0008477.

[43] N. L. Biggs, LSE report LSE-CDAM-00-04 (May 2000).

[44] N. L. Biggs and P. Reinfeld, LSE report LSE-CDAM-00-07 (June 2000), http://www.cdam.lse.ac.uk/Reports/.

[45] S.-C. Chang and R. Shrock, Physica A, in press (cond-mat/0007491).

[46] The differences include the chromatic polynomial, the singular locus, and the question of whether there are chromatic zeros with $\operatorname{Re}(q)<0$. We are informed by Prof. Biggs and P. Reinfeld that they now agree with our calculation and conclusions and are revising LSECDAM-00-07 accordingly. We thank Prof. N. Biggs and P. Reinfeld for correspondence on this. 